\title{
Generating functions for permutations which contain a given descent set
}

\author{
Jeffrey Remmel \\ Department of Mathematics \\ University of California, San Diego \\ La Jolla, CA 92093-0112. USA \\ jremmel@ucsd.edu
}

\author{
Manda Riehl \\ Department of Mathematics \\ University of Wisconsin, Eau Claire \\ Eau Claire, WI, 54702. USA \\ riehlar@uwec.edu
}

Submitted: Jan 30, 2009; Accepted: Feb 7, 2010; Published: Feb 15, 2010

Mathematics Subject Classification: 05A15, 68R15, 06A07

\begin{abstract}
A large number of generating functions for permutation statistics can be obtained by applying homomorphisms to simple symmetric function identities. In particular, a large number of generating functions involving the number of descents of a permutation $\sigma, \operatorname{des}(\sigma)$, arise in this way. For any given finite set $S$ of positive integers, we develop a method to produce similar generating functions for the set of permutations of the symmetric group $S_{n}$ whose descent set contains $S$. Our method will be to apply certain homomorphisms to symmetric function identities involving ribbon Schur functions.

Keywords: ribbon Schur functions, descent sets, generating functions, permutation statistics
\end{abstract}

\section{Introduction}

There has been a long line of research, [2], [3], [1], [8], [9], [12], [13], [14], [16], [11], which shows that a large number of generating functions for permutation statistics can be obtained by applying homomorphisms defined on the ring of symmetric functions $\Lambda$ to simple symmetric function identities. For example, the $n$-th elementary symmetric function, $e_{n}$ and the $n$-th homogeneous symmetric function, $h_{n}$, are defined by the generating functions

$$
E(t)=\sum_{n \geqslant 0} e_{n} t^{n}=\prod_{i}\left(1+x_{i} t\right)
$$

and

$$
H(t)=\sum_{n \geqslant 0} h_{n} t^{n}=\prod_{i} \frac{1}{1-x_{i} t} .
$$


We let $P(t)=\sum_{n \geqslant 0} p_{n} t^{n}$ where $p_{n}=\sum_{i} x_{i}^{n}$ is the $n$-th power symmetric function. For any partition, $\mu=\left(\mu_{1}, \ldots, \mu_{\ell}\right)$, we let $h_{\mu}=\prod_{i=1}^{\ell} h_{\mu_{i}}, e_{\mu}=\prod_{i=1}^{\ell} e_{\mu_{i}}$, and $p_{\mu}=\prod_{i=1}^{\ell} p_{\mu_{i}}$. Now it is well known that

$$
H(t)=1 / E(-t)
$$

and

$$
P(t)=\frac{\sum_{n \geqslant 1}(-1)^{n-1} n e_{n} t^{n}}{E(-t)} .
$$

A surprisingly large number of results on generating functions for various permutation statistics that have appeared in the literature as well as a large number of new generating functions for permutation statistics can be derived by applying ring homomorphisms defined on $\Lambda$ to simple symmetric function identities such as (1.1) and (1.2).

Let $S_{n}$ denote the symmetric group and write $\sigma \in S_{n}$ in one line notation as $\sigma=$ $\sigma_{1} \ldots \sigma_{n}$. In this section, we shall consider the following statistics on $S_{n}$.

$$
\begin{array}{ll}
\operatorname{Des}(\sigma)=\left\{i: \sigma_{i}>\sigma_{i+1}\right\} & \operatorname{Rise}(\sigma)=\left\{i: \sigma_{i}<\sigma_{i+1}\right\} \\
\operatorname{des}(\sigma)=|\operatorname{Des}(\sigma)| & \operatorname{rise}(\sigma)=|\operatorname{Rise}(\sigma)| \\
\operatorname{inv}(\sigma)=\sum_{i<j} \chi\left(\sigma_{i}>\sigma_{j}\right) & \operatorname{coinv}(\sigma)=\sum_{i<j} \chi\left(\sigma_{i}<\sigma_{j}\right)
\end{array}
$$

where for any statement $A, \chi(A)=1$ if $A$ is true and $\chi(A)=0$ if $A$ is false. Also if $\alpha^{1}, \ldots, \alpha^{k} \in S_{n}$, then we shall write comdes $\left(\alpha^{1}, \ldots, \alpha^{k}\right)=\left|\bigcap_{i=1}^{k} \operatorname{Des}\left(\alpha^{i}\right)\right|$. We should also note that these definitions make sense for any sequence $\sigma=\sigma_{1} \cdots \sigma_{n}$ of natural numbers. We shall also use standard notation for $q$-analogues. That is, we let

$$
\begin{gathered}
{[n]_{q}=1+q+\cdots+q^{n-1}=\frac{1-q^{n}}{1-q},} \\
{[n]_{q} !=[n]_{q}[n-1]_{q} \cdots[1]_{q},} \\
{\left[\begin{array}{c}
n \\
k
\end{array}\right]_{q}=\frac{[n]_{q} !}{[k]_{q} ![n-k]_{q} !} \text {, and }} \\
{\left[\begin{array}{c}
n \\
\left.\lambda_{1}, \ldots, \lambda_{\ell}\right]_{q}=\frac{[n]_{q} !}{\left[\lambda_{1}\right]_{q} ! \cdots\left[\lambda_{\ell}\right]_{q} !} .
\end{array}\right.}
\end{gathered}
$$

Similarly, we can define $(p, q)$-analogues of these formulas by replacing $[n]_{q}$ by

$$
[n]_{p, q}=p^{n-1}+p^{n-2} q+\cdots+p^{1} q^{n-2}+q^{n-1}=\frac{p^{n}-q^{n}}{p-q} .
$$

Then the following results can be proved by applying a suitable homomorphism to the identity (1.1).

1) $\sum_{n=0}^{\infty} \frac{u^{n}}{n !} \sum_{\sigma \in S_{n}} x^{\operatorname{des}(\sigma)}=\frac{1-x}{-x+e^{u(x-1)}}$.

2) (Carlitz 1970) [4]

$\sum_{n=0}^{\infty} \frac{u^{n}}{(n !)^{2}} \sum_{(\sigma, \tau) \in S_{n} \times S_{n}} x^{\operatorname{comdes}(\sigma, \tau)}=\frac{1-x}{-x+J(u(x-1))}$. 
3) (Stanley 1979) [15]

$\sum_{n=0}^{\infty} \frac{u^{n}}{[n] !} \sum_{\sigma \in S_{n}} x^{\operatorname{des}(\sigma)} q^{i n v(\sigma)}=\frac{1-x}{-x+e_{q}(u(x-1))}$.

4) (Stanley 1979) [15]

$\sum_{n=0}^{\infty} \frac{u^{n}}{[n] !} \sum_{\sigma \in S_{n}} x^{\operatorname{des}(\sigma)} q^{\operatorname{coinv}(\sigma)}=\frac{1-x}{-x+E_{q}(u(x-1))}$.

5) (Fedou and Rawlings 1995) [7]

$\sum_{n=0}^{\infty} \frac{u^{n}}{[n]_{q} ![n]_{p} !} \sum_{(\sigma, \tau) \in S_{n} \times S_{n}} x^{\operatorname{comdes}(\sigma, \tau)} q^{i n v(\sigma)} p^{i n v(\tau)}=\frac{1-x}{-x+J_{q, p}(u(x-1))}$.

Here $J(u)=\sum_{n \geqslant 0} \frac{u^{n}}{n ! n !}, e_{q}(u)=\sum_{n=0}^{\infty} \frac{u^{n}}{[n]_{q} !} q^{\left(\begin{array}{c}n \\ 2\end{array}\right)}, E_{q}(u)=\sum_{n=0}^{\infty} \frac{u^{n}}{[n]_{q} !}$, and

$J_{q, p}(u)=\sum_{n=0}^{\infty} \frac{u^{n}}{[n]_{q} ![n]_{p} !} q^{\left(\begin{array}{c}n \\ 2\end{array}\right)} p^{\left(\begin{array}{c}n \\ 2\end{array}\right)}$.

Langley and Remmel [9] proved a common generalization of all these results. To state the Langley-Remmel result, we first need to establish some notation. If $\boldsymbol{\Sigma}=$ $\left(\sigma^{(1)}, \ldots, \sigma^{(L)}\right)$ is a sequence of permutations in $S_{n}$, then we define

$$
\operatorname{Comdes}(\boldsymbol{\Sigma})=\left(\bigcap_{i=1}^{L} \operatorname{Des}\left(\sigma^{(i)}\right)\right)
$$

and

$$
\operatorname{comdes}(\boldsymbol{\Sigma})=|\operatorname{Comdes}(\boldsymbol{\Sigma})| \text {. }
$$

If $\mathbf{Q}=\left(q_{1}, \ldots, q_{L}\right)$ and $\mathbf{P}=\left(p_{1}, \ldots, p_{L}\right)$, then, for any $m \geqslant 1$, we let

$$
\begin{aligned}
& \mathbf{Q}^{m}=q_{1}^{m} \cdots q_{L}^{m}, \quad \mathbf{P}^{m}=p_{1}^{m} \cdots p_{L}^{m}, \\
& {[n]_{\mathbf{Q}}=\prod_{i=1}^{L}[n]_{q_{i}}, \quad[n]_{\mathbf{P}, \mathbf{Q}}=\prod_{i=1}^{L}[n]_{p_{i}, q_{i}},} \\
& {[n]_{\mathbf{Q}} !=\prod_{i=1}^{L}[n]_{q_{i}} !, \quad[n]_{\mathbf{P}, \mathbf{Q}} !=\prod_{i=1}^{L}[n]_{p_{i}, q_{i}} !,} \\
& {\left[\begin{array}{c}
n \\
\lambda_{1}, \ldots, \lambda_{k}
\end{array}\right]_{\mathrm{Q}}=\prod_{i=1}^{L}\left[\begin{array}{c}
n \\
\lambda_{1}, \ldots, \lambda_{k}
\end{array}\right]_{q_{i}},} \\
& {\left[\begin{array}{c}
n \\
\lambda_{1}, \ldots, \lambda_{k}
\end{array}\right]_{\mathbf{P}, \mathbf{Q}}=\prod_{i=1}^{L}\left[\begin{array}{c}
n \\
\lambda_{1}, \ldots, \lambda_{k}
\end{array}\right]_{p_{i}, q_{i}},} \\
& \mathbf{Q}^{i n v(\boldsymbol{\Sigma})}=\prod_{i=1}^{L} q_{i}^{i n v\left(\sigma^{(i)}\right)} \text {, and } \\
& \mathbf{P}^{\operatorname{coinv}(\mathbf{\Sigma})}=\prod_{i=1}^{L} p_{i}^{\operatorname{coinv}\left(\sigma^{(i)}\right)}
\end{aligned}
$$


Generalizing $J_{q, p}(u)$, we define

$$
\exp (t, \mathbf{Q}, \mathbf{P})=\sum_{n \geqslant 0} \frac{t^{n} \mathbf{Q}^{\left(\begin{array}{c}
n \\
2
\end{array}\right)}}{[\mathbf{n}]_{\mathbf{P}, \mathbf{Q}} !} .
$$

Then Langley and Remmel [9] proved that for all $L \geqslant 1$,

$$
\sum_{n=0}^{\infty} \frac{t^{n}}{[n]_{\mathbf{P}, \mathbf{Q} !}} \sum_{\boldsymbol{\Sigma}=\left(\sigma^{(1)}, \ldots, \sigma^{(L)}\right) \in S_{n}^{L}} x^{\operatorname{comdes}(\boldsymbol{\Sigma})} \mathbf{Q}^{i n v(\boldsymbol{\Sigma})} \mathbf{P}^{\operatorname{coinv}(\boldsymbol{\Sigma})}=\frac{1-x}{-x+\exp (t, \mathbf{Q}, \mathbf{P})}
$$

The main goal of this paper is to find a uniform way to compute similar generating functions where we sum over $\sigma$ that $S \subseteq \operatorname{Des}(\sigma)$ where $S$ is any finite subset of $\{1,2, \ldots\}$. That is, for any finite set $S \subseteq\{1,2, \ldots\}$, we shall show how to compute the following generating function:

$$
\begin{aligned}
F_{S}^{L}(x, \mathbf{Q}, \mathbf{P}) & =F_{S}^{L}\left(x, q_{1}, \ldots, q_{L}, p_{1}, \ldots, p_{L}\right) \\
& =\sum_{n \geqslant 0} \frac{t^{n}}{[n]_{\mathbf{P}, \mathbf{Q}} !} \sum_{\boldsymbol{\Sigma} \in S_{n}^{L}, S \subseteq \operatorname{Comdes}(\boldsymbol{\Sigma})} x^{\operatorname{comdes}(\boldsymbol{\Sigma})} \mathbf{Q}^{\operatorname{inv}(\boldsymbol{\Sigma})} \mathbf{P}^{\operatorname{coinv}(\boldsymbol{\Sigma})} .
\end{aligned}
$$

The outline of this paper is as follows. In section 2, we shall supply the necessary background on symmetric functions and the combinatorics of the entries of the transition matrices between various bases of symmetric functions that we need for our developments. In section 3, we shall derive our key identity involving ribbon Schur functions which will be used to derive our expression for $F_{S}^{L}(x, \mathbf{Q}, \mathbf{P})$. In section 4 , we shall give our method for finding the generating function for $F_{S}^{L}(x, \mathbf{Q}, \mathbf{P})$ and give some examples. Finally in section 5, we shall discuss some extensions of our results for $F_{S}^{L}(x, \mathbf{Q}, \mathbf{P})$ where instead of considering generating functions where we sum over $\boldsymbol{\Sigma}$ such that $S \subseteq \operatorname{Comdes}(\boldsymbol{\Sigma})$, we consider generating functions where we sum over $\boldsymbol{\Sigma}$ such that $S \subseteq \operatorname{Comdes}(\boldsymbol{\Sigma})$ and $T \nsubseteq$ Comdes $(\boldsymbol{\Sigma})$ where $S$ and $T$ are a pair finite disjoint sets.

\section{Symmetric functions and transition matrices}

In this section, we shall present the background on symmetric functions and the combinatorics of the transition matrices between various bases of symmetric functions that will be needed for our methods.

Let $\Lambda_{n}$ denote the space of homogeneous symmetric functions of degree $n$ over infinitely many variables $x_{1}, x_{2}, \ldots$. We say that $\lambda=\left(0<\lambda_{1} \leqslant \cdots \leqslant \lambda_{k}\right)$ is a partition of $n$, written $\lambda \vdash n$, if $\lambda_{1}+\cdots+\lambda_{k}=n=|\lambda|$. We let $\ell(\lambda)=k$ be the number of parts of $\lambda$. It is well known that $\left\{h_{\lambda}: \lambda \vdash n\right\},\left\{e_{\lambda}: \lambda \vdash n\right\}$, and $\left\{p_{\lambda}: \lambda \vdash n\right\}$ are all bases of $\Lambda_{n}$, see [10].

We let $F_{\lambda}$ denote the Ferrers diagram of $\lambda$. If $\mu=\left(\mu_{1}, \ldots, \mu_{m}\right)$ is a partition where $m \leqslant k$ and $\lambda_{i} \geqslant \mu_{i}$ for all $i \leqslant m$, we let $F_{\lambda / \mu}$ denote the skew shape that results by removing the cells of $F_{\mu}$ from $F_{\lambda}$. For example, Figure 1 pictures the skew diagram 
$(1,2,3,3) /(1,2)$ on the left. We let $|\lambda / \mu|$ denote the number of squares in $\lambda / \mu$. A columnstrict tableau $T$ of shape $\lambda / \mu$ is any filling of $F_{\lambda / \mu}$ with natural numbers such that entries in each row are weakly increasing from left to right, and entries in each column are strictly increasing from bottom to top. We define the weight of $T$ to be $w(T)=x_{1}^{\alpha_{1}} x_{2}^{\alpha_{2}} \cdots$ where $\alpha_{i}$ is the number of times that $i$ occurs in $T$. For example, on the right of Figure 1, we have pictured a column strict tableau of shape $(1,2,3,3) /(1,2)$ and weight $x_{1}^{2} x_{2} x_{3} x_{4}^{2}$. Then the skew Schur function indexed by $\lambda / \mu$ is given by $s_{\lambda / \mu}=\sum_{T} w(T)$, where the sum runs over all column strict tableaux of shape $\lambda / \mu$. We define a ribbon (or zigzag)
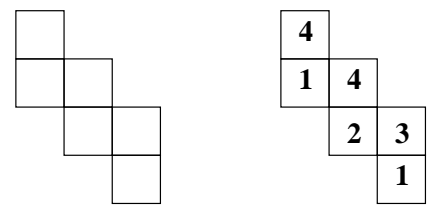

Figure 1: The skew Ferrers diagram and column strict tableau of shape $(1,2,3,3) /(1,2)$.

shape to be a connected skew shape that contains no $2 \times 2$ array of boxes. Ribbon (or zigzag) Schur functions are the skew Schur functions with a ribbon shape and are indexed by compositions. A composition $\beta=\left(\beta_{1}, \ldots, \beta_{k}\right)$ of $n$, denoted $\beta \models n$, is a sequence of positive integers such that $\beta_{1}+\beta_{2}+\cdots+\beta_{k}=n$. Given a composition $\beta=\left(\beta_{1}, \ldots, \beta_{k}\right)$, we let $Z_{\beta}$ denote the skew Schur function corresponding to the zigzag shape whose row lengths are $\beta_{1}, \ldots, \beta_{k}$ reading from top to bottom. For example, Figure 2 shows the zigzag shape corresponding to the composition $(2,3,1,4)$. We let $\lambda(\beta)$ denote the partition that arises from $\beta$ by arranging its parts in weakly increasing order and $\ell(\beta)$ denote the number of parts of $\beta$. For example, if $\beta=(2,3,1,2)$, then $\lambda(\beta)=(1,2,2,3)$. We also define $\operatorname{shape}(\beta)=\lambda / \nu$ such that $F_{\beta}=F_{\lambda / \nu}$.

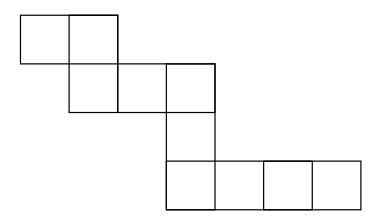

Figure 2: The ribbon shape corresponding to the composition $(2,3,1,4)$, so that $s_{(2,4,4,7) /(1,3,3)}=Z_{(2,3,1,4)}$.

A rim hook of $\lambda$ is a connected sequence of cells, $h$, along the northeast boundary of $F_{\lambda}$ which has a ribbon shape and is such that if we remove $h$ from $F_{\lambda}$, we are left with the Ferrers diagram of another partition. More generally, $h$ is a rim hook of a skew shape $\lambda / \mu$ if $h$ is a rim hook of $\lambda$ which does not intersect $\mu$. We say that $h$ is a special rim hook of $\lambda / \mu$ if $h$ starts in the cell which occupies the north-west corner of $\lambda / \mu$. We say that $h$ is a transposed special rim hook of $\lambda / \mu$ if $h$ ends in the cell which occupies the south-east corner of $\lambda / \mu$.

A special rim hook tabloid (transposed special rim hook tabloid) of shape $\lambda / \mu$ and type $\nu, T$, is a sequence of partitions $T=\left(\mu=\lambda^{(0)} \subset \lambda^{(1)} \subset \cdots \lambda^{(k)}=\lambda\right)$, such that 
for each $1 \leqslant i \leqslant k, \lambda^{(i)} / \lambda^{(i-1)}$ is a special rim hook (transposed special rim hook) of $\lambda^{(i)}$ such that the weakly increasing rearrangement of $\left(\left|\lambda^{(1)} / \lambda^{(0)}\right|, \cdots,\left|\lambda^{(k)} / \lambda^{(k-1)}\right|\right)$ is equal to $\nu$. We show an example of a special rim hook tabloid and a transposed special rim hook tabloid of shape $(4,5,6,6) /(1,3,3)$ in Figure 3 . We define the sign of a special rim

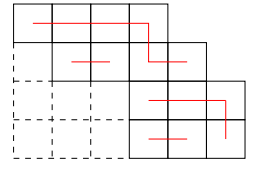

A Special Rim Hook Tableau of shape $(4,5,6,6) /(1,3,3)$ and

type $(2,2,4,6)$

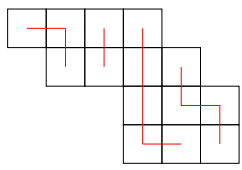

A Transposed Special Rim Hook Tableau of shape $(4,5,6,6) /(1,3,3)$ and type $(2,3,4,5)$

Figure 3: A special rim hook tabloid and a transposed special rim hook tabloid.

hook $h_{i}=\lambda^{(i)} / \lambda^{(i-1)}$ to be $\operatorname{sgn}\left(h_{i}\right)=(-1)^{r\left(h_{i}\right)-1}$, where $r\left(h_{i}\right)$ is the number of rows that $h_{i}$ occupies. Likewise, we define the sign of a transposed special rim hook to be $t$-sgn $\left(h_{i}\right)=(-1)^{c\left(h_{i}\right)-1}$, where $c\left(h_{i}\right)$ is the number of columns that $h_{i}$ occupies. Let $\operatorname{SRHT}(\nu, \lambda / \mu)(t-S R H T(\nu, \lambda / \mu))$ equal the set of special rim hook tabloids (transposed special rim hook tabloids) of type $\nu$ and shape $\lambda / \mu$. If $T \in S R H T(\nu, \lambda / \mu)$, we let $\operatorname{sgn}(T)=\prod_{H \in T} \operatorname{sgn}(H)$. If $T \in t-S R H T(\nu, \lambda / \mu)$, then $t-\operatorname{sgn}(T)=\prod_{H \in T} t-\operatorname{sgn}(H)$. For $|\lambda / \mu|=|\nu|$, we let

$$
\begin{aligned}
K_{\nu, \lambda / \mu}^{-1} & =\sum_{T \in S R H T(\nu, \lambda / \mu)} \operatorname{sgn}(T) \quad \text { and } \\
T K_{\nu, \lambda / \mu}^{-1} & =\sum_{T \in t-S R H T(\nu, \lambda / \mu)} \operatorname{sgn}(T) .
\end{aligned}
$$

Then Ĕgecioğlu and Remmel [5] proved that

$$
s_{\lambda / \mu}=\sum_{\nu} K_{\nu, \lambda / \mu}^{-1} h_{\nu} \quad \text { and } \quad s_{\lambda / \mu}=\sum_{\nu} T K_{\nu, \lambda / \mu}^{-1} e_{\nu}
$$

Eğecioğlu and Remmel [6] also proved that

$$
h_{\mu}=\sum_{\lambda \vdash n}(-1)^{n-\ell(\lambda)} B_{\lambda, \mu} e_{\lambda}
$$

where $B_{\lambda, \mu}$ is the number of $\lambda$-brick tabloids of shape $\mu$. Here a $\lambda$-brick tabloid $T$ of shape $\mu$ is a filling of $F_{\mu}$ with bricks of sizes corresponding to the parts of $\lambda$ such that (i) no two bricks overlap and (ii) each brick lies within a single row. For example, the $(1,1,2,2)$-brick tabloids of shape $(2,4)$ are pictured in Figure 4 . More generally, let $\mathcal{B}_{\lambda, \mu}$ denote the set of $\lambda$-brick tabloids of shape $\mu=\left(\mu_{1}, \ldots, \mu_{k}\right)$.

Next we introduce a class of symmetric functions $p_{\lambda}^{\vec{u}}$ that were first introduced in [9] and [12]. Suppose that $R$ is a ring and we are given any sequence $\vec{u}=\left(u_{1}, u_{2}, \ldots\right)$ of elements of $R$. Then for any brick tabloid $T \in \mathcal{B}_{\lambda, \mu}$, we let $\left(b_{1}, \ldots, b_{k}\right)$ denote the lengths 

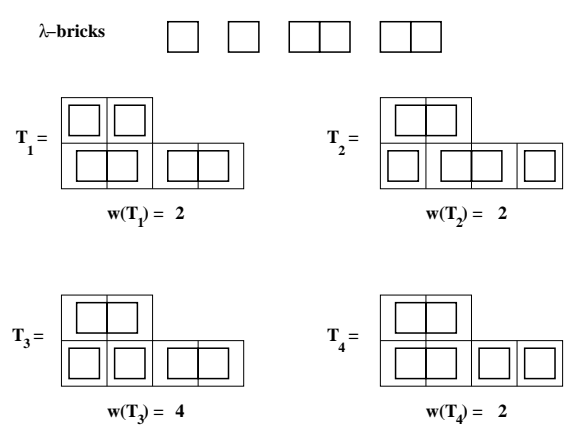

Figure 4: $B_{\lambda, \mu}$ and $w\left(B_{\lambda, \mu}\right)$ for $\lambda=(1,1,2,2)$ and $\mu=(2,4)$.

of the bricks which lie at the right end of the rows of $T$ reading from top to bottom and we set $w_{\vec{u}}(T)=u_{b_{1}} \cdots u_{b_{k}}$. We then set $w_{\vec{u}}\left(B_{\lambda, \mu}\right)=\sum_{T \in \mathcal{B}_{\lambda, \mu}} w_{\vec{u}}(T)$. For example if $u=(1,2,3, \ldots)$, then $w_{\vec{u}}(T)=w(T)$ is just the product of the lengths of the bricks that lie at the end of the rows of $T$. We have given $w(T)$ for each of the brick tabloids in Figure 4. We can now define the family of symmetric functions $p_{\lambda}^{\vec{u}}$ as follows. First, we let $p_{0}^{\vec{u}}=1$ and

$$
p_{n}^{\vec{u}}=\sum_{\lambda \vdash n}(-1)^{n-\ell(\lambda)} w_{\vec{u}}\left(B_{\lambda,(n)}\right) e_{\lambda}
$$

for $n \geqslant 1$. Finally, if $\mu=\left(\mu_{1}, \ldots, \mu_{k}\right)$ is a partition of $n$, we set $p_{\mu}^{\vec{u}}=p_{\mu_{1}}^{\vec{u}} \cdots p_{\mu_{k}}^{\vec{u}}$. We note that it follows from results of Eğecioğlu and Remmel $[6]$ that if $u=(1,2,3, \ldots)$, then $p_{n}^{\vec{u}}$ is just the usual power symmetric function $p_{n}$. Thus we call $p_{n}^{\vec{u}}$ a generalized power symmetric function.

Mendes and Remmel $[13,12]$ proved the following:

$$
\begin{aligned}
\sum_{n \geqslant 1} p_{n}^{\vec{u}} t^{n} & =\frac{\sum_{n \geqslant 1}(-1)^{n-1} u_{n} e_{n} t^{n}}{E(-t)} \text { and } \\
1+\sum_{n \geqslant 1} p_{n}^{\vec{u}} t^{n} & =\frac{1+\sum_{n \geqslant 1}(-1)^{n}\left(e_{n}-u_{n} e_{n}\right) t^{n}}{E(-t)}
\end{aligned}
$$

Note if we take $\vec{u}=(1,1, \ldots)$, then $(2.3)$ becomes

$$
1+\sum_{n \geqslant 1} p_{n}^{\vec{u}} t^{n}=1+\frac{\sum_{n \geqslant 1}(-1)^{n-1} e_{n} t^{n}}{\sum_{n \geqslant 0}(-1)^{n} e_{n} t^{n}}=\frac{1}{\sum_{n \geqslant 0}(-1)^{n} e_{n} t^{n}}=1+\sum_{n \geqslant 1} h_{n} t^{n}
$$

which implies $p_{n}^{(1,1, \ldots)}=h_{n}$.

Other special cases for $\vec{u}$ give well-known generating functions. By taking $u_{n}=$ $(-1)^{k} \chi(n \geqslant k+1)$ for some $k \geqslant 1, p_{n}^{\vec{u}}$ is the Schur function corresponding to the partition $\left(1^{k}, n\right)$. 


\section{$3 \quad$ An identity for ribbon Schur functions}

Let $\alpha=\left(\alpha_{k}, \alpha_{k-1}, \ldots, \alpha_{1}\right)$ be a composition. Then we let $\alpha^{(0)}=\alpha, \alpha^{(k)}=\emptyset$ and $\alpha^{(j)}=\left(\alpha_{k}, \ldots, \alpha_{j+1}\right)$ for $j=1, \ldots, k-1$. For example, if $\alpha=(3,2,1,3)$, then $\alpha^{(0)}=$ $(3,2,1,3), \alpha^{(1)}=(3,2,1), \alpha^{(2)}=(3,2), \alpha^{(3)}=(3)$, and $\alpha^{(4)}=\emptyset$. We let $(\alpha, n)$ denote the composition that results by adding an extra part of size $n$ at the end of $\alpha$, i.e. $(\alpha, n)=\left(\alpha_{k}, \alpha_{k-1}, \ldots, \alpha_{1}, n\right)$. Let $Z_{\emptyset}=1$.

The main goal of this section is to prove the following identity for ribbon Schur functions.

Theorem 3.1.

$$
\begin{aligned}
\sum_{n \geqslant 1} Z_{(\alpha, n)} t^{n+|\alpha|}= & \frac{\sum_{j=0}^{k}(-1)^{j} Z_{\alpha^{(j)}} t^{\left|\alpha^{(j)}\right|}}{E(-t)}+ \\
& (-1)^{k-1}+\sum_{j=1}^{k}(-1)^{j-1} \sum_{r=1}^{\alpha_{j}-1} Z_{\left(\alpha^{(j)}, r\right)} t^{r+\left|\alpha^{(j)}\right|} .
\end{aligned}
$$

For example, suppose $\alpha=(3,2,1,3)$. Then Theorem 3.1 becomes

$$
\begin{aligned}
\sum_{n \geqslant 1} Z_{(\alpha, n)} t^{n+|\alpha|}= & \frac{Z_{(3,2,1,3)} t^{9}-Z_{(3,2,1)} t^{6}+Z_{(3,2)} t^{5}-Z_{(3)} t^{3}+1}{E(-t)} \\
& -1+\left(Z_{(3,2,1,2)} t^{8}+Z_{(3,2,1,1)} t^{7}\right)+\left(Z_{(3,1)} t^{4}\right)-\left(Z_{(2)} t^{2}+Z_{(1)} t\right) .
\end{aligned}
$$

This example helps explain how to think of the right-hand side of (3.1). The numerator of the term $\frac{\sum_{j=0}^{k}(-1)^{j} Z_{\alpha(j)} t^{\mid \alpha^{(j)}} \mid}{E(-t)}$ is just the alternating sum of the $Z_{\alpha^{(j)}} t^{\left|\alpha^{(j)}\right|}$ 's where the first term $Z^{\alpha} t^{|\alpha|}=Z_{\alpha^{(0)}} t^{\left|\alpha^{(0)}\right|}$ starts with a plus sign. For each $1 \leqslant j \leqslant k-1$, the ribbon shapes that appear in $\sum_{r=1}^{\alpha_{j}-1} Z_{\left(\alpha^{(j)}, r\right)} t^{r+\left|\alpha^{(j)}\right|}$ consist of the ribbon shapes that one can obtain from the ribbon shape corresponding to $\left(\alpha_{k}, \ldots, \alpha_{j+1}, \alpha_{j}\right)$ by removing at least one, but not all, of the squares at the end of the last row. We call these the auxiliary ribbon shapes derived from $\alpha^{(j-1)}$. In our example, if we start with the ribbon shape $\alpha^{(0)}=(3,2,1,3)$ as pictured in the top of Figure 5, then the auxiliary ribbon shapes derived from $\alpha^{(0)}$ are the two ribbon shapes pictured at the bottom of Figure 5. Note that if $\alpha_{j}=1$, then there are no auxiliary shapes derived from $\alpha^{(j-1)}$. Thus the second term in (3.1) consists of alternating signs of the generating functions of ribbon Schur functions indexed by the auxiliary shapes derived from the $\alpha^{(j-1)}$ 's for $j=1, \ldots, k$. Moreover, the term $(-1)^{k-1}$ which appears at the start of the second term can be thought of as the term which would be derived from the ribbon shape $\alpha^{(k-1)}$, which is just a single row $\left(\alpha_{k}\right)$, by removing all the squares, leaving $Z_{\emptyset}=1$.

We should also note that in the special case where $\alpha=\left(1^{k}\right)$, there are no auxiliary 

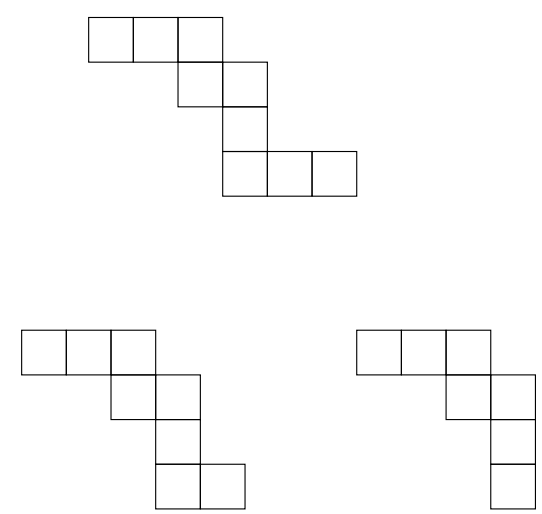

Figure 5: The auxiliary ribbon shapes derived from the ribbon shape $(3,2,1,3)$.

shapes, so we obtain

$$
\begin{aligned}
\sum_{n \geqslant 1} s_{\left(1^{k}, n\right)} t^{k+n} & =\frac{\sum_{j=0}^{k}(-1)^{j} Z_{\left(1^{k-j}\right)} t^{k-j}}{E(-t)}+(-1)^{k-1} \\
& =\frac{(-1)^{k} \sum_{j=0}^{k}(-1)^{j} e_{j} t^{j}}{E(-t)}-\frac{(-1)^{k} E(-t)}{E(-t)} \\
& =\frac{-(-1)^{k} \sum_{j \geqslant k+1}(-1)^{j} e_{j} t^{j}}{E(-t)} \\
& =\frac{\sum_{j \geqslant k+1}(-1)^{j-1}(-1)^{k} e_{j} t^{j}}{E(-t)} .
\end{aligned}
$$

This is just the special case of $(2.3)$ when $u_{n}=(-1)^{k} \chi(n \geqslant k+1)$, since $p_{n}^{(-1)^{k} \chi(n \geqslant k+1)}$ is the Schur function corresponding to the partition $\left(1^{k}, n\right)$.

\section{Proof of Theorem 3.1.}

Proof. We start with the expansion $s_{\lambda / \mu}=\sum_{\nu} K_{\nu, \lambda / \mu}^{-1} h_{\nu}$. If $\lambda / \mu$ corresponds to the ribbon shape $\alpha=\left(\alpha_{k}, \ldots, \alpha_{1}\right)$, then we can classify the special rim hook tabloids by the length of the last special rim hook. For example, a typical special rim hook in the case where $\alpha=(3,2,4,5,3)$ is pictured in Figure 6 . Since in a special rim hook tabloid each of the rim hooks must start on the left hand border, it follows that the rim hook which ends in the lower-most square must cover the last $j$ rows for some $j \in\{1, \ldots, k\}$. Now suppose that $H$ is the last rim hook pictured in Figure 6. We consider the sum $\sum_{\mu} \sum_{T \in F(\mu, H)} \operatorname{sgn}(T) h_{\mu}$ where $F(\mu, H)$ is the set of special rim hook tabloids of type $\mu$ and ribbon shape $\alpha=(3,2,4,5,3)$ such that the last special rim hook of $T$ is $H$. Since the filling of the rim hooks in the first three rows of ribbon shape $\alpha=(3,2,4,5,3)$ is arbitrary, this sum will equal

$$
\operatorname{sgn}(H) h_{|H|} \sum_{\nu \vdash 9} \sum_{T \in S R H T(\nu, \gamma / \delta)} \operatorname{sgn}(T) h_{\nu}
$$


where $\gamma / \delta$ is just the skew shape corresponding to the ribbon shape $(3,2,4)$. So this sum is just $\operatorname{sgn}(H) h_{|H|} Z_{(3,2,4)}$.

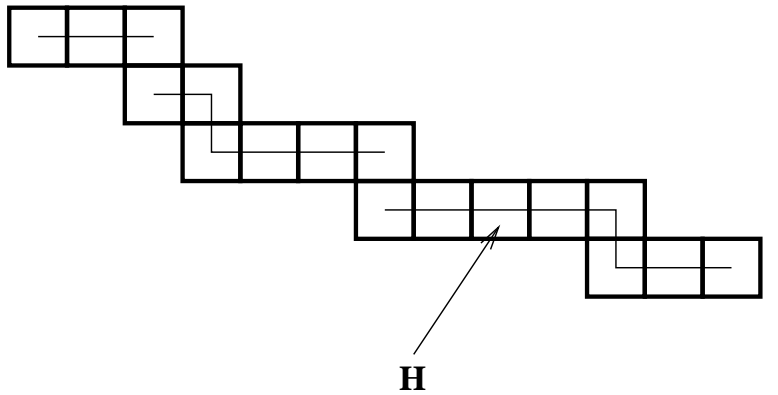

Figure 6: A special rim hook tabloid of the ribbon shape $(3,2,4,5,3)$.

It follows that if we classify the special rim hook tabloids $T$ of the ribbon shape $(\alpha, n)$ by the number $j$ of rows in the ribbon shape corresponding to $\alpha$ that the last rim hook of $T$ covers, then we obtain

$$
Z_{(\alpha, n)}=\sum_{j=0}^{k}(-1)^{j} Z_{\alpha^{(j)}} h_{n+\alpha_{1}+\cdots+\alpha_{j}} .
$$

Thus

$$
\begin{aligned}
\sum_{n \geqslant 1} Z_{(\alpha, n)} t^{n+|\alpha|}= & \sum_{j=0}^{k}(-1)^{j} Z_{\alpha^{(j)}} t^{\left|\alpha^{(j)}\right|} \sum_{n \geqslant 1} h_{n+\alpha_{1}+\cdots+\alpha_{j}} t^{n+\alpha_{1}+\cdots+\alpha_{j}} \\
= & \sum_{j=0}^{k}(-1)^{j} Z_{\alpha^{(j)}} t^{\left|\alpha^{(j)}\right|}\left(H(t)-\sum_{r=0}^{\alpha_{1}+\cdots+\alpha_{j}} h_{r} t^{r}\right) \\
= & \sum_{j=0}^{k}(-1)^{j} Z_{\alpha^{(j)}} t^{\left|\alpha^{(j)}\right|}\left(\frac{1}{E(-t)}-\sum_{r=0}^{\alpha_{1}+\cdots+\alpha_{j}} h_{r} t^{r}\right) \\
= & \frac{\sum_{j=0}^{k}(-1)^{j} Z_{\alpha^{(j)}} t^{|\alpha|}}{E(-t)}- \\
& \left(Z_{\alpha} t^{|\alpha|}+\sum_{j=1}^{k}(-1)^{j} Z_{\alpha^{(j)}} t^{\left|\alpha^{(j)}\right|} \sum_{r=0}^{\alpha_{1}+\cdots+\alpha_{j}} h_{r} t^{r}\right) .
\end{aligned}
$$

Now consider the sum

$$
Z_{\alpha} t^{|\alpha|}+\sum_{j=1}^{k}(-1)^{j} Z_{\alpha^{(j)}} t^{\left|\alpha^{(j)}\right|} \sum_{r=0}^{\alpha_{1}+\cdots+\alpha_{j}} h_{r} t^{r} .
$$

Combining the $r=0$ term in the sum with $Z_{\alpha} t^{|\alpha|}$, we obtain

$$
\sum_{j=0}^{k}(-1)^{j} Z_{\alpha^{(j)}} t^{\left|\alpha^{(j)}\right|}+\sum_{j=1}^{k}(-1)^{j} Z_{\alpha^{(j)}} t^{\left|\alpha^{(j)}\right|} \sum_{r=1}^{\alpha_{1}+\cdots+\alpha_{j}} h_{r} t^{r} \text {. }
$$


It is a consequence of the Littlewood-Richardson rule that for any composition $\beta=$ $\left(\beta_{t}, \ldots, \beta_{1}\right)$,

$$
Z_{\beta} h_{r}=Z_{(\beta, r)}+Z_{\left(\beta_{t}, \ldots, \beta_{2}, \beta_{1}+r\right)} .
$$

Thus we see that (3.3) is equal to

$$
\sum_{j=0}^{k}(-1)^{j} Z_{\alpha^{(j)}} t^{\left|\alpha^{(j)}\right|}+\sum_{j=1}^{k} \sum_{r=1}^{\alpha_{1}+\cdots+\alpha_{j}}(-1)^{j} t^{\left|\alpha^{(j)}\right|+r}\left(Z_{\left(\alpha_{k}, \ldots, \alpha_{j+1}, r\right)}+Z_{\left(\alpha_{k}, \ldots, \alpha_{j+2}, \alpha_{j+1}+r\right)}\right) .
$$

We can organize the $Z_{\beta}$ 's that appear in (3.4) by the number of parts, $s$, of $\beta$.

For $s=0$, there is one term: $(-1)^{k} Z_{\alpha^{(k)}}=(-1)^{k}$.

For $1 \leqslant s<k$, we obtain the terms

$$
\begin{aligned}
& \left.(-1)^{k-s} Z_{\left(\alpha_{k}, \ldots, \alpha_{k-s+1}\right.}\right)^{\left|\alpha^{(k-s)}\right|}+ \\
& (-1)^{k-s+1} \sum_{r=1}^{\alpha_{1}+\cdots+\alpha_{k-s+1}} Z_{\left(\alpha_{k}, \ldots, \alpha_{k-s+2}, r\right)} t^{\alpha_{k}+\cdots+\alpha_{k-s+2}+r}+ \\
& (-1)^{k-s} \sum_{r=1}^{\alpha_{1}+\cdots+\alpha_{k-s}} Z_{\left(\alpha_{k}, \ldots, \alpha_{k-s+2}, \alpha_{k-s+1}+r\right)} t^{\alpha_{k}+\cdots+\alpha_{k-s+1}+r} \\
& \left.=(-1)^{k-s+1} \sum_{r=1}^{\alpha_{k-s+1}-1} Z_{\left(\alpha_{k}, \ldots, \alpha_{k-s+2}, r\right.}\right)^{\alpha_{k}+\cdots+\alpha_{k-s+2}+r} .
\end{aligned}
$$

For $s=k$, we have the terms

$$
Z_{\left(\alpha_{k}, \ldots, \alpha_{1}\right)} t^{|\alpha|}-\sum_{r=1}^{\alpha_{1}} Z_{\alpha_{k}, \ldots, \alpha_{2}, r} t^{\alpha_{k}+\ldots \alpha_{2}+r}=-\sum_{r=1}^{\alpha_{1}-1} Z_{\alpha_{k}, \ldots, \alpha_{2}, r} t^{\alpha_{k}+\ldots \alpha_{2}+r} .
$$

Combining these cases together, we see that (3.3) is

$$
(-1)^{k}+\sum_{j=1}(-1)^{j} \sum_{r=1}^{\alpha_{j}-1} Z_{\left(\alpha^{(j)}, r\right)} t^{\left|\alpha^{(j)}\right|+r} .
$$

Combining (3.5) with (3.2) yields (3.1).

\section{Methods for Computing Generating Functions}

In this section, we shall describe how we can use ribbon Schur functions to compute various generating functions over sets of permutations which contain a given descent set. 
In particular, we shall give methods to compute

$$
\begin{aligned}
F_{S}^{L}(x, \mathbf{Q}, \mathbf{P}) & =F_{S}^{L}\left(x, q_{1}, \ldots, q_{L}, p_{1}, \ldots, p_{L}\right) \\
& =\sum_{n \geqslant 0} \frac{t^{n}}{[n]_{\mathbf{P}, \mathbf{Q}} !} \sum_{\substack{\boldsymbol{\Sigma} \in S_{n}^{L} \\
S \subseteq \operatorname{Comdes}(\boldsymbol{\Sigma})}} x^{\operatorname{comdes}(\boldsymbol{\Sigma})} \mathbf{Q}^{i n v(\boldsymbol{\Sigma})} \mathbf{P}^{\operatorname{coinv}(\boldsymbol{\Sigma})}
\end{aligned}
$$

The method proceeds in three steps. First, for any composition $\alpha=\left(\alpha_{1}, \ldots, \alpha_{k}\right)$, of $n$, define $h_{\alpha}=h_{\alpha_{1}} \cdots h_{\alpha_{k}}$ and

$$
\operatorname{Set}(\alpha)=\left\{\alpha_{1}, \alpha_{1}+\alpha_{2}, \ldots, \alpha_{1}+\cdots+\alpha_{k-1}\right\} .
$$

Then for $\sigma \in S_{n}$, we define

$$
\begin{aligned}
\operatorname{Des}_{\alpha}(\sigma) & =\operatorname{Des}(\sigma)-\operatorname{Set}(\alpha), \\
\operatorname{des}_{\alpha}(\sigma) & =\left|\operatorname{Des}_{\alpha}(\sigma)\right|, \\
\operatorname{Rise}_{\alpha}(\sigma) & =\operatorname{Rise}_{(\sigma)} \cup \operatorname{Set}(\alpha), \text { and } \\
\operatorname{rise}_{\alpha}(\sigma) & =\left|\operatorname{Rise}_{\alpha}(\sigma)\right| .
\end{aligned}
$$

If $\boldsymbol{\Sigma}=\left(\sigma_{1}, \ldots, \sigma_{L}\right) \in S_{n}^{L}$, then we let

$$
\begin{aligned}
\operatorname{Comdes}_{\alpha}(\boldsymbol{\Sigma}) & =\bigcap_{i=1}^{L} \operatorname{Des}_{\alpha}\left(\sigma_{i}\right) \\
\operatorname{comdes}_{\alpha}(\boldsymbol{\Sigma}) & =\left|\operatorname{Comdes}_{\alpha}(\boldsymbol{\Sigma})\right| \\
\text { oneRise }_{\alpha}(\boldsymbol{\Sigma}) & =\{1, \ldots, n\}-\operatorname{Comdes}_{\alpha}(\boldsymbol{\Sigma}), \text { and } \\
\text { onerise }_{\alpha}(\boldsymbol{\Sigma}) & =\mid \text { oneRise }_{\alpha}(\boldsymbol{\Sigma}) \mid .
\end{aligned}
$$

Define a ring homomorphism $\xi$ from the ring of symmetric functions $\Lambda$ to the polynomial ring $Q\left(q_{1}, \ldots, q_{L}, p_{1}, \ldots, p_{L}\right)[x]$ by setting

$$
\xi\left(e_{n}\right)=\frac{(1-x)^{n-1} \mathbf{Q}^{\left(\begin{array}{c}
n \\
2
\end{array}\right)}}{[n]_{\mathbf{P}, \mathbf{Q}} !} .
$$

This ring homomorphism was used by Langley and Remmel [9] to prove (1.4).

Then our first step is to prove the following result which is a simple modification of the proof that Langley and Remmel [9] used to prove (1.4) that results by using the method of Beck and Remmel [1] to determine the image of $h_{\lambda}$ under ring homomorphisms.

Theorem 4.1. For any composition $\alpha=\left(\alpha_{1}, \ldots, \alpha_{k}\right)$ of $n$,

$$
[n]_{\mathbf{P}, \mathbf{Q}} ! \xi\left(h_{\alpha}\right)=\sum_{\boldsymbol{\Sigma}=\left(\sigma_{1}, \ldots, \sigma_{L}\right) \in S_{n}^{L}} x^{\operatorname{comdes}_{\alpha}(\Sigma)} \mathbf{Q}^{i n v(\boldsymbol{\Sigma})} \mathbf{P}^{\operatorname{coinv}(\boldsymbol{\Sigma})}
$$



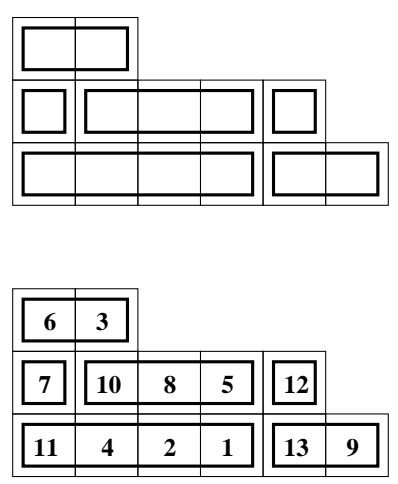

Figure 7: The brick tabloid $T=(2,1,3,1,4,2)$ in $\mathcal{B}_{\left(1^{2}, 2^{2}, 3,4\right),(2,5,6)}$.

Proof. First we consider the case where $\alpha$ is a partition of $n$. Given a brick tabloid $T \in \mathcal{B}_{\mu, \alpha}$, let $b_{1}, \ldots, b_{\ell(\mu)}$ be the sequence which records the lengths of the bricks in $T$ where we read the rows from top to bottom and bricks in the rows from left to right in each row. In such a situation, we shall write $T=\left(b_{1}, \ldots, b_{\ell(\mu)}\right)$. For example, for the brick tabloid $T \in \mathcal{B}_{\left(1^{2}, 2^{2}, 3,4\right),(2,5,6)}$ pictured at the top of Figure 7 , we would write $T=(2,1,3,1,4,2)$.

Then we know that

$$
\begin{aligned}
{[n]_{\mathbf{P}, \mathbf{Q}} ! \xi\left(h_{\alpha}\right) } & =[n]_{\mathbf{P}, \mathbf{Q}} ! \sum_{\mu \vdash n}(-1)^{n-\ell(\mu)} B_{\mu, \alpha} \xi\left(e_{\mu}\right) \\
& =[n]_{\mathbf{P}, \mathbf{Q}} ! \sum_{\mu \vdash n}(-1)^{n-\ell(\mu)} \sum_{T=\left(b_{1}, \ldots, b_{\ell(\mu)}\right) \in \mathcal{B}_{\mu, \alpha}} \prod_{i=1}^{\ell(\mu)} \frac{(1-x)^{b_{i}-1} \mathbf{Q}^{\left(\begin{array}{c}
b_{i} \\
2
\end{array}\right)}}{\left[b_{i}\right]_{\mathbf{P}, \mathbf{Q}} !} \\
& =\sum_{\mu \vdash n T=\left(b_{1}, \ldots, b_{\ell(\mu)}\right) \in \mathcal{B}_{\mu, \alpha}}\left[\begin{array}{c}
n \\
b_{1}, \ldots, b_{\ell(\mu)}
\end{array}\right]_{\mathbf{P}, \mathbf{Q}} \mathbf{Q}^{\sum_{i=1}^{\ell(\mu)}\left(\begin{array}{c}
b_{i} \\
2
\end{array}\right)(x-1)^{n-\ell(\mu)}} .
\end{aligned}
$$

Fix a brick tabloid $T=\left(b_{1}, \ldots, b_{\ell(\mu)}\right) \in B_{\mu, n}$. We want to give a combinatorial interpretation to $\left.\left[\begin{array}{c}n \\ b_{1}, \ldots, b_{\ell(\mu)}\end{array}\right]\right]_{\mathbf{P}, \mathbf{Q}}$. Let $D F(T)$ denote the set of all fillings of the cells of $T$ with the numbers $1, \ldots, n$ so that the numbers decrease within each brick reading from left to right. We think of each such filling as a permutation of $S_{n}$ by reading the numbers in rows from top to bottom and the numbers from left to right in each row. For example, the filled brick tabloid at the bottom of Figure 7 is an element of $D F((2,1,3,1,4,2))$, with corresponding permutation 63710851211421139 . Then we have the following lemma.

Lemma 4.2. Let $T=\left(b_{1}, \ldots, b_{\ell(\mu)}\right)$ be a brick tabloid in $\mathcal{B}_{\mu, \alpha}$. Then

$$
q^{\sum_{i}\left(\begin{array}{c}
b_{i} \\
2
\end{array}\right)}\left[\begin{array}{c}
n \\
b_{1}, \ldots, b_{\ell(\mu)}
\end{array}\right]_{p, q}=\sum_{\sigma \in D F(T)} q^{i n v(\sigma)} p^{\operatorname{coinv}(\sigma)} .
$$


Proof. It follows from a result of Carlitz [4] that for positive integers $b_{1}, \ldots, b_{\ell}$ which sum to $n$,

$$
\left[\begin{array}{c}
n \\
b_{1}, \ldots, b_{\ell}
\end{array}\right]_{p, q}=\sum_{r \in \mathcal{R}\left(1^{\left.b_{1}, \ldots, \ell^{b} \ell\right)}\right.} q^{i n v(r)} p^{\operatorname{coinv}(r)}
$$

where $\mathcal{R}\left(1^{b_{1}}, \ldots, \ell^{b_{\ell}}\right)$ is the set of rearrangements of $b_{1} 1^{\prime}$ 's, $b_{2} 2$ 's, etc. Consider a rearrangement $r$ of $1^{b_{1}}, \ldots, \ell^{b_{\ell}}$ and construct a permutation $\sigma_{r}$ by labeling the 1 's from right to left with $1,2, \ldots, b_{1}$, the 2 's from right to left with $b_{1}+1, \ldots, b_{1}+b_{2}$, and in general the $i$ 's from right to left with $1+\sum_{j=1}^{i-1} b_{j}, \ldots, b_{i}+\sum_{j=1}^{i-1} b_{j}$. In this way, $\sigma_{r}^{-1}$ starts with the positions of the 1's in decreasing order, followed by the positions of the 2's in $r$ in decreasing order, etc. For example, if $T=(2,1,3,1,4,2) \in \mathcal{B}_{\left(1^{2}, 2^{2}, 3,4\right),(2,5,6)}$ is the brick tabloid pictured at the top of Figure 7, then one possible rearrangement to consider is $r=5515312363546$. Below we display $\sigma_{r}$ and $\sigma_{r}^{-1}$.

\begin{tabular}{lcccccccccccccc} 
& & 1 & 2 & 3 & 4 & 5 & 6 & 7 & 8 & 9 & 10 & 11 & 12 & 13 \\
\hline$r$ & $=$ & 5 & 5 & 1 & 5 & 3 & 1 & 2 & 3 & 6 & 3 & 5 & 4 & 6 \\
$\sigma_{r}$ & $=$ & 11 & 10 & 2 & 9 & 6 & 1 & 3 & 5 & 13 & 4 & 8 & 7 & 12 \\
$\sigma_{r}^{-1}$ & $=$ & 6 & 3 & 7 & 10 & 8 & 5 & 12 & 11 & 4 & 2 & 1 & 13 & 9.
\end{tabular}

It is then easy to see that

$$
\begin{aligned}
& \left(\begin{array}{l}
2 \\
2
\end{array}\right)+\left(\begin{array}{l}
1 \\
2
\end{array}\right)+\left(\begin{array}{l}
2 \\
2
\end{array}\right)+\left(\begin{array}{l}
1 \\
2
\end{array}\right)+\left(\begin{array}{l}
4 \\
2
\end{array}\right)+\left(\begin{array}{l}
2 \\
2
\end{array}\right)+i n v(r)=\operatorname{inv}\left(\sigma_{r}\right)=\operatorname{inv}\left(\sigma_{r}^{-1}\right) \text { and } \\
& \operatorname{coinv}(r)=\operatorname{coinv}\left(\sigma_{r}\right)=\operatorname{coinv}\left(\sigma_{r}^{-1}\right) .
\end{aligned}
$$

We can think of $\sigma_{r}^{-1}$ as a filling of the cells of the brick tabloid $T=(2,1,3,4,2)$ with the numbers $1, \ldots, 13$ such that the numbers within each brick are decreasing, reading from left to right. In fact, this filling is precisely the filling pictured at the bottom of Figure 7.

Thus in general, for any $T=\left(b_{1}, \ldots, b_{\ell(\mu)}\right) \in \mathcal{B}_{\mu, \alpha}$, the correspondence which takes $r \in \mathcal{R}\left(1^{b_{1}}, \ldots, \ell^{b_{\ell}}\right)$ to $\sigma_{r}^{-1}$ shows that

$$
q^{\sum\left(\begin{array}{c}
b_{i} \\
2
\end{array}\right)}\left[\begin{array}{c}
n \\
b_{1}, \ldots, b_{\ell(\mu)}
\end{array}\right]_{p, q}=\sum_{\sigma \in D F(T)} q^{i n v(\sigma)} p^{\operatorname{coinv}(\sigma)}
$$

as desired.

It follows that for any $T=\left(b_{1}, \ldots, b_{\ell(\mu)}\right) \in \mathcal{B}_{\mu, \alpha}$,

$$
\begin{aligned}
& \mathbf{Q}^{\sum_{i=1}^{\ell(\mu)}\left(\begin{array}{c}
b_{i} \\
2
\end{array}\right)}\left[\begin{array}{c}
n \\
b_{1}, \ldots, b_{\ell(\mu)}
\end{array}\right]_{\mathbf{P}, \mathbf{Q}}=\prod_{i=1}^{L} q_{i}^{\sum\left(\begin{array}{c}
b_{i} \\
2
\end{array}\right)}\left[\begin{array}{c}
n \\
b_{1}, \ldots, b_{\ell(\mu)}
\end{array}\right]_{p_{i}, q_{i}} \\
& =\prod_{i=1}^{L} \sum_{\sigma^{(i)} \in D F(T)} q_{i}^{i n v\left(\sigma^{(i)}\right)} p_{i}^{\operatorname{coinv}\left(\sigma^{(i)}\right)} .
\end{aligned}
$$

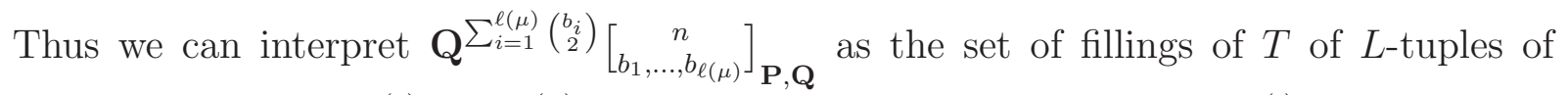
permutations $\boldsymbol{\Sigma}=\left(\sigma^{(1)}, \ldots, \sigma^{(L)}\right)$ such that for each $i$, the elements of $\sigma^{(i)}$ are decreasing 
within each brick of $T$, and we weight such a filling with $\mathbf{Q}^{i n v(\boldsymbol{\Sigma})} \mathbf{P}^{\operatorname{coinv}(\boldsymbol{\Sigma})}$. For example, if $T=(2,2,3,2,4,3) \in \mathcal{B}_{\left(2^{3}, 3^{2}, 4\right),(4,5,7)}$ and $L=3$, then such a filling of $T$ is pictured in Figure 8 . We can then interpret the term $(x-1)^{n-\ell(\mu)}$ as taking such a filling and labeling each cell which is not at the end of a brick with either $x$ or -1 , and labeling each cell at the end of a brick with 1. Again, we have pictured such a labeling of the cells of $T$ in Figure 8. We shall call such an object $\mathcal{O}$ a labeled filled brick tabloid. We define the weight of $\mathcal{O}, W(\mathcal{O})$, to be product over all the labels of the cells times $\mathbf{Q}^{i n v(\boldsymbol{\Sigma})} \mathbf{P}^{\operatorname{coinv}(\boldsymbol{\Sigma})}$ if $T$ is filled with permutations $\boldsymbol{\Sigma}=\left(\sigma^{(1)}, \ldots \sigma^{(L)}\right)$. Thus for the object pictured in Figure 8 ,

$$
W(\mathcal{O})=(-1)^{4} x^{6} q_{1}^{i n v\left(\sigma^{(1)}\right)} q_{2}^{\operatorname{inv}\left(\sigma^{(2)}\right)} q_{3}^{i n v\left(\sigma^{(3)}\right)} p_{1}^{\operatorname{coinv}\left(\sigma^{(1)}\right)} p_{2}^{\operatorname{coinv}\left(\sigma^{(2)}\right)} p_{3}^{\operatorname{coinv}\left(\sigma^{(3)}\right)} .
$$

\begin{tabular}{|c|c|c|c|c|c|c|}
\hline$\underline{x}$ & 1 & -1 & 1 & & & \\
\hline 6 & 2 & 16 & 10 & & & \\
\hline 10 & 6 & 12 & 4 & & & \\
\hline 16 & 4 & 8 & 2 & & & \\
\hline $\mathbf{x}$ & $\mathbf{x}$ & 1 & $\mathbf{x}$ & & & \\
\hline 14 & 12 & 7 & 13 & 4 & & \\
\hline 16 & 13 & 3 & 7 & 2 & & \\
\hline 15 & 9 & 7 & 14 & 11 & & \\
\hline $\mathbf{x}$ & -1 & -1 & 1 & -1 & $\mathbf{x}$ & 1 \\
\hline 15 & 5 & 3 & 1 & 11 & 9 & 8 \\
\hline 9 & 8 & 5 & 1 & 15 & 14 & 11 \\
\hline 13 & 10 & 5 & 3 & 12 & 6 & 1 \\
\hline
\end{tabular}

$$
\begin{aligned}
& \sigma^{(1)}=62161014127134155311198 \\
& \sigma^{(2)}=10612416133729851151411 \\
& \sigma^{(3)}=16482159714111310531261
\end{aligned}
$$

Figure 8: A labeled filled brick tabloid of shape $(4,5,7)$.

We let $\mathcal{L} F(\alpha)$ denote the set of all objects that can be created in this way from brick tabloids $T$ of shape $\alpha$. It follows that

$$
[n]_{\mathbf{P}, \mathbf{Q}} ! \xi\left(h_{\alpha}\right)=\sum_{\mathcal{O} \in \mathcal{L} F(\alpha)} W(\mathcal{O}) .
$$

Next we define an involution $I: \mathcal{L} F(\alpha) \rightarrow \mathcal{L} F(\alpha)$. Given $\mathcal{O} \in \mathcal{L} F(\alpha)$, read the cells of $\mathcal{O}$ in the same order that we read the underlying permutations and look for the first cell $c$ such that either:

(i) $c$ is labeled with -1 or

(ii) $c$ is at the end of end of brick $b$, the cell $c+1$ is immediately to the right of $c$ and starts another brick $b^{\prime}$, and each permutation $\sigma^{(i)}$ decreases from $c$ to $c+1$.

If we are in case $(\mathrm{i})$, then $I(\mathcal{O})$ is the labeled filled brick tabloid which is obtained from $\mathcal{O}$ by splitting the brick $b$ that contains $c$ into two bricks $b_{1}$ and $b_{2}$, where $b_{1}$ contains 
the cells of $b$ up to and including the cell $c$ and $b_{2}$ contains the remaining cells of $b$, and changing the label on $c$ from -1 to 1 . In case (ii), $I(\mathcal{O})$ is the labeled filled brick tabloid which is obtained from $\mathcal{O}$ by combining the two brick $b$ and $b^{\prime}$ into a single brick and changing the label on cell $c$ from 1 to -1 . Finally, if neither case (i) or case (ii) applies, then we let $I(\mathcal{O})=\mathcal{O}$. For example, if $\mathcal{O}$ is the labeled filled brick tabloid pictured in Figure 8, then $I(\mathcal{O})$ is pictured in Figure 9.

\begin{tabular}{|c|c|c|c|c|c|c|}
\hline$\underline{\mathbf{x}}$ & 1 & -1 & 1 & & & \\
\hline $\begin{array}{l}6 \\
10 \\
16\end{array}$ & $\begin{array}{l}2 \\
6 \\
4\end{array}$ & $\begin{array}{l}16 \\
12 \\
8\end{array}$ & $\begin{array}{l}10 \\
4 \\
2\end{array}$ & & & \\
\hline $\mathbf{x}$ & $\mathbf{x}$ & 1 & $\mathbf{x}$ & & & \\
\hline 14 & 12 & 7 & 13 & 4 & & \\
\hline 16 & 13 & 3 & 7 & 2 & & \\
\hline 15 & 9 & 7 & 14 & 11 & & \\
\hline$\underline{\mathbf{x}}$ & 1 & -1 & 1 & -1 & $\mathbf{x}$ & 1 \\
\hline 15 & 5 & 3 & 1 & 11 & 9 & 8 \\
\hline 9 & 8 & 5 & 1 & 15 & 14 & 11 \\
\hline 13 & 10 & 5 & 3 & 12 & 6 & 1 \\
\hline
\end{tabular}

$$
\begin{aligned}
& \sigma^{(1)}=62161014127134155311198 \\
& \sigma^{(2)}=10612416133729851151411 \\
& \sigma^{(3)}=16482159714111310531261
\end{aligned}
$$

Figure 9: $I(\mathcal{O})$.

It is easy to see that if $I(\mathcal{O}) \neq \mathcal{O}$, the $W(I(\mathcal{O}))=-W(\mathcal{O})$ since we change the label on cell $c$ from 1 to -1 or vice versa. Moreover, it is easy to check that $I^{2}$ is the identity. Thus $I$ shows that

$$
\begin{aligned}
{[n]_{\mathbf{P}, \mathbf{Q}} ! \xi\left(h_{\alpha}\right) } & =\sum_{\mathcal{O} \in \mathcal{L} F(\alpha)} W(\mathcal{O}) \\
& =\sum_{\mathcal{O} \in \mathcal{L} F(\alpha), I(\mathcal{O})=\mathcal{O}} W(\mathcal{O}) .
\end{aligned}
$$

Thus we must examine the fixed points of $I$. Clearly if $I(\mathcal{O})=\mathcal{O}$, then $\mathcal{O}$ can have no cells which are labeled with -1 . Also it must be the case that between any two consecutive bricks in the same row of $\mathcal{O}$, at least one of the underlying permutations $\sigma^{(i)}$ must increase. It follows that each cell $c$ which is not at the end of the brick in $\mathcal{O}$ is labeled with $x$ and each of the permutations $\sigma^{(i)}$ has a descent at $c$ so that $c \in \operatorname{Comdes}(\boldsymbol{\Sigma})$. All the other cells of $\mathcal{O}$ are either at the end of a brick which has another brick to its right in which case $c \notin \operatorname{Comdes}(\boldsymbol{\Sigma})$ or $c$ is at the end of a row in which case $c \in \operatorname{Set}(\alpha)$. All such cells have label 1, so that $W(\mathcal{O})=x^{\operatorname{comdes}_{\alpha}(\boldsymbol{\Sigma})} \mathbf{Q}^{\operatorname{inv}(\boldsymbol{\Sigma})} \mathbf{P}^{\operatorname{coinv}(\boldsymbol{\Sigma})}$.

Now, if we are given $\boldsymbol{\Sigma}=\left(\sigma^{(1)}, \ldots, \sigma^{(L)}\right) \in S_{n}^{L}$, we can construct a fixed point of $I$ from $\boldsymbol{\Sigma}$ by using $\left(\sigma^{(1)}, \ldots, \sigma^{(L)}\right)$ to fill a tabloid of shape $\alpha$, then drawing the bricks so 
that the cells $c$ which end bricks are precisely the elements of oneRise $(\boldsymbol{\Sigma}) \cup \operatorname{Set}(\alpha)$. This shows that

$$
\sum_{\mathcal{O} \in \mathcal{L} F(\alpha), I(\mathcal{O})=\mathcal{O}} W(\mathcal{O})=\sum_{\boldsymbol{\Sigma} \in S_{n}^{L}} x^{\operatorname{comdes}_{\alpha}(\sigma)} \mathbf{Q}^{i n v(\boldsymbol{\Sigma})} \mathbf{P}^{\operatorname{coinv}(\boldsymbol{\Sigma})}
$$

as desired.

Now suppose $\beta$ is an arbitrary composition which can be rearranged to the partition $\alpha$. Observe that the order in which we read the rows of the brick tabloid $T \in \mathcal{B}_{\mu, \alpha}$ determined how we read the bricks and how we associated a sequence of permutations with a filled brick tabloid. That is, we ordered the rows $R_{1}, \ldots, R_{\ell(\alpha)}$ of $T$ by reading the rows from top to bottom. This, in turn, determined how we ordered the bricks so that we could write $T=\left(b_{1}, \ldots, b_{\ell(\mu)}\right)$ and determined how we associated a permutation with each labeled filled brick tabloid based on $T$. That is, we ordered the bricks by reading the bricks from left to right in each row and reading the rows in the order $R_{1}, \ldots, R_{\ell(\alpha)}$. Similarly, we read the cells in rows from left to right and we read the rows in order $R_{1}, \ldots, R_{\ell(\alpha)}$ to determine how we associated a permutation with a filled brick tabloid. Now suppose that we decide to order the rows as $R_{\tau_{1}}, \ldots, R_{\tau_{\ell(\alpha)}}$ for some permutation $\tau \in S_{\ell(\alpha)}$ such that $\left(\left|R_{\tau_{1}}\right|, \ldots,\left|R_{\tau_{\ell(\alpha)}}\right|\right)=\beta$, where $\left|R_{i}\right|$ denotes the length of row $R_{i}$. Then we would have a new way to order the bricks by reading the bricks from left to right in each row and reading the rows in order $R_{\tau_{1}}, \ldots, R_{\tau_{\ell(\alpha)}}$. Similarly, we would have a new way to associate a permutation with each labeled filled brick tabloid $T$ of shape $\alpha$ by reading the cells in rows from left to right and reading the rows in order $R_{\tau_{1}}, \ldots, R_{\tau_{\ell(\alpha)}}$. Everything in the proof will be exactly as before, except that cells at the end of the rows $R_{\tau_{1}}, \ldots, R_{\tau_{\ell(\alpha)}}$ would correspond to the positions in $\operatorname{Set}(\beta)$ rather than $\operatorname{Set}(\alpha)$. In this way, we could show that

$$
[n]_{\mathbf{P}, \mathbf{Q}} ! \xi\left(h_{\alpha}\right)=\sum_{\boldsymbol{\Sigma}=\left(\sigma_{1}, \ldots, \sigma_{L}\right) \in S_{n}^{L}} x^{\operatorname{comdes}_{\beta}(\sigma)} \mathbf{Q}^{i n v(\boldsymbol{\Sigma})} \mathbf{P}^{\operatorname{coinv}(\boldsymbol{\Sigma})}
$$

for any composition $\beta$ that rearranges to $\alpha$. Since $h_{\beta}=h_{\alpha}$ in this case, it follows that (4.1) holds for any composition $\alpha$.

Next, given a composition of $n, \alpha=\left(\alpha_{1}, \ldots, \alpha_{k}\right)$, let $F_{\alpha}$ denote the ribbon shape corresponding to $\alpha$ and $Z_{\alpha}$ denote the ribbon Schur function corresponding to $\alpha$. Then we have the following.

\section{Theorem 4.3.}

$$
[n]_{\mathbf{P}, \mathbf{Q}} ! \xi\left(Z_{\alpha}\right)=\frac{(1-x)^{k-1}}{x^{k-1}} \sum_{\substack{\boldsymbol{\Sigma}=\left(\sigma^{(1)}, \ldots, \sigma^{(L)}\right) \in S_{n}^{L} \\ \operatorname{Set}(\alpha) \subseteq \operatorname{Comdes}(\boldsymbol{\Sigma})}} x^{\operatorname{comdes}(\boldsymbol{\Sigma})} \mathbf{Q}^{\operatorname{inv}(\boldsymbol{\Sigma})} \mathbf{P}^{\operatorname{coinv}(\boldsymbol{\Sigma})} .
$$

Proof. We can expand the $Z_{\alpha}$ using using (2.1) as

$$
Z_{\alpha}=S_{\lambda / \nu}=\sum_{\mu \vdash|\lambda / \nu|} h_{\mu} \sum_{T \in S R H T(\mu, \lambda / \nu)} \operatorname{sgn}(T) .
$$


Applying $\xi$ to both sides of (4.2), we obtain

$$
\begin{aligned}
{[n]_{\mathbf{P}, \mathbf{Q}} ! \xi\left(Z_{\alpha}\right) } & =[n]_{\mathbf{P}, \mathbf{Q}} ! \sum_{\mu \vdash n} \xi\left(h_{\mu}\right) \sum_{T \in S R H T(\mu, \lambda / \nu)} \operatorname{sgn}(T) \\
& =\sum_{T \in S R H T(\operatorname{shape}(\alpha))} \operatorname{sgn}(T)[n]_{\mathbf{P}, \mathbf{Q} ! \xi\left(h_{\beta(T)}\right)} \\
& =\sum_{T \in S R H T(\operatorname{shape}(\alpha))} \operatorname{sgn}(T) \sum_{\boldsymbol{\Sigma} \in S_{n}^{L}} x^{\operatorname{comdes}_{\beta(T)}(\boldsymbol{\Sigma})} \mathbf{Q}^{\operatorname{inv}(\boldsymbol{\Sigma})} \mathbf{P}^{\operatorname{coinv}(\boldsymbol{\Sigma})}
\end{aligned}
$$

where $\beta(T)$ is the composition induced by reading the rim hooks in $T$ from top to bottom. Thus we can identify $[n]_{\mathbf{P}, \mathbf{Q}} ! \xi\left(Z_{\alpha}\right)$ with a sum over filled special rim hook tabloids $F(T)$, where each cell of the underlying special rim hook tabloid is filled with an $L$-tuple of numbers in such a way that when we read the numbers in cells starting from the top, we get a sequence of permutations $\boldsymbol{\Sigma}_{F(T)}=\left(\sigma_{F(T)}^{(1)}, \ldots, \sigma_{F(T)}^{(L)}\right) \in S_{n}^{L}$. For example, consider the filled special rim tabloid $F\left(T_{1}\right)$ pictured in the top left of Figure 10 . Then $L=2$, $\alpha=(3,1,3,2), \beta(T)=(4,5)$, and the underlying pair of permutations $\Sigma=\left(\sigma^{(1)}, \sigma^{(2)}\right)$ is

$$
\begin{aligned}
\sigma^{(1)} & =593768421 \\
\sigma^{(2)} & =641285379 .
\end{aligned}
$$

In this case Comdes $(\boldsymbol{\Sigma})=\{2,6\}$ so we have put $x$ 's on top of each of the cells. The weight of this configuration is then

$$
\operatorname{sgn}(T) x^{\operatorname{comdes}(\boldsymbol{\Sigma})} \mathbf{Q}^{i n v(\boldsymbol{\Sigma})} \mathbf{P}^{\operatorname{coinv}(\boldsymbol{\Sigma})}=x^{2} q_{1}^{26} q_{2}^{12} p_{1}^{10} p_{2}^{24} .
$$
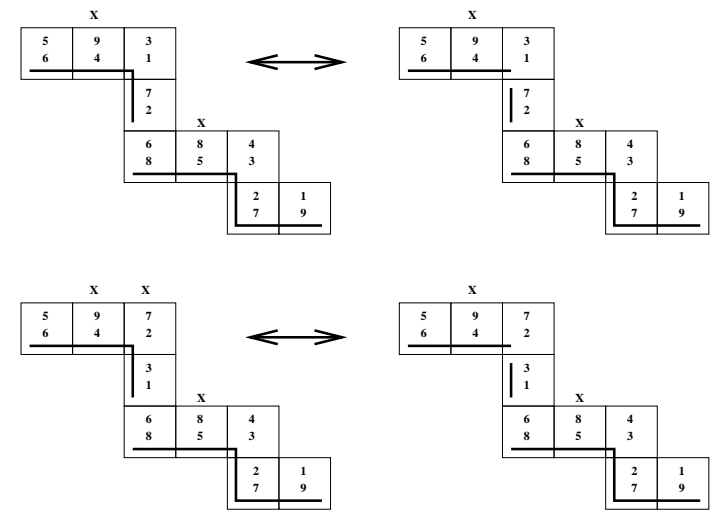

Figure 10: Pairing of special rim hook tabloids of shape $F_{(3,1,3,2)}$.

Let first us examine the fillings in pairs with identical integer fillings and identical special rim hooks, except that one has a break in the special rim hooks at the first row and the other does not. For example, if $F\left(T_{2}\right)$ is the filled special rim hook tabloid at the top right of Figure 10, then $\left(F\left(T_{1}\right), F\left(T_{2}\right)\right)$ is such a pair. Similarly, if $F\left(T_{3}\right)$ is the filled 
special rim hook tabloid at the bottom left of Figure 10 and $F\left(T_{4}\right)$ is the filled special rim hook tabloid at the bottom right of Figure 10, then $\left(F\left(T_{3}\right), F\left(T_{4}\right)\right)$ is such a pair.

Now suppose that $(F(T), F(S))$ is any such pair of special rim hook tabloids where the first rim hook of $S$ ends in the first row and the first rim hook of $T$ does not end the first row. It is easy to see that the sign of the underlying special rim hook tabloid $T$ is -1 raised to number of vertical segments that are part of rim hooks, so that $\operatorname{sgn}(T)=-\operatorname{sgn}(S)$. Let $c=\alpha_{1}$ be the cell at the end of the first row $F_{\alpha}$. If $c \notin \operatorname{Comdes}(\boldsymbol{\Sigma})$, then $c$ is in neither $\operatorname{Comdes}_{\beta}(S)$ nor $\operatorname{Comdes}_{\beta}(T)$ so that

$$
x^{\operatorname{comdes}_{\beta(T)}(\boldsymbol{\Sigma})} \mathbf{Q}^{i n v(\boldsymbol{\Sigma})} \mathbf{P}^{\operatorname{coinv}(\boldsymbol{\Sigma})}=x^{\operatorname{comdes}_{\beta(S)}(\boldsymbol{\Sigma})} \mathbf{Q}^{i n v(\boldsymbol{\Sigma})} \mathbf{P}^{\operatorname{coinv}(\boldsymbol{\Sigma})}
$$

Hence any two such filled special rim hook tabloids will contribute 0 to

$$
\sum_{T \in S R H T(\operatorname{shape}(\alpha))} \operatorname{sgn}(T) \sum_{\boldsymbol{\Sigma} \in S_{n}^{L}} x^{\operatorname{comdes}_{\beta(T)}(\boldsymbol{\Sigma})} \mathbf{Q}^{\operatorname{inv}(\boldsymbol{\Sigma})} \mathbf{P}^{\operatorname{coinv}(\boldsymbol{\Sigma})} .
$$

For example, the pair $\left(F\left(T_{1}\right), F\left(T_{2}\right)\right)$ pictured at the top of Figure 10 is such a pair. However if $c \in \operatorname{Comdes}(\boldsymbol{\Sigma})$, then $c \in \operatorname{Comdes}_{\beta}(T)$, but $c \notin \operatorname{Comdes}_{\beta}(S)$. In this case, it follows that

$$
\operatorname{sgn}(T) x^{\operatorname{comdes}_{\beta(T)}(\boldsymbol{\Sigma})} \mathbf{Q}^{i n v(\boldsymbol{\Sigma})} \mathbf{P}^{\operatorname{coinv}(\boldsymbol{\Sigma})}=(-x) \operatorname{sgn}(S) x^{\operatorname{comdes}_{\beta(S)}(\boldsymbol{\Sigma})} \mathbf{Q}^{\operatorname{inv}(\boldsymbol{\Sigma})} \mathbf{P}^{\operatorname{coinv}(\boldsymbol{\Sigma})} .
$$

For example, the pair $\left(F\left(T_{3}\right), F\left(T_{4}\right)\right)$ pictured at the bottom of Figure 10 is such a pair. In this situation, the pair $(F(T), F(S))$ will contribute

$$
(1-x) \operatorname{sgn}(S) x^{\operatorname{comdes}_{\beta(S)}(\boldsymbol{\Sigma})} \mathbf{Q}^{i n v(\boldsymbol{\Sigma})} \mathbf{P}^{\operatorname{coinv}(\boldsymbol{\Sigma})}
$$

to the sum

$$
\sum_{T \in S R H T(\operatorname{shape}(\alpha))} \operatorname{sgn}(T) \sum_{\boldsymbol{\Sigma} \in S_{n}^{L}} x^{\operatorname{comdes}_{\beta(T)}(\boldsymbol{\Sigma})} \mathbf{Q}^{\operatorname{inv}(\boldsymbol{\Sigma})} \mathbf{P}^{\operatorname{coinv}(\boldsymbol{\Sigma})} .
$$

It follows that we can replace the sum

$$
\sum_{T \in S R H T(\operatorname{shape}(\alpha))} \operatorname{sgn}(T) \sum_{\boldsymbol{\Sigma} \in S_{n}^{L}} x^{\operatorname{comdes}_{\beta(T)}(\boldsymbol{\Sigma})} \mathbf{Q}^{\operatorname{inv}(\boldsymbol{\Sigma})} \mathbf{P}^{\operatorname{coinv}(\boldsymbol{\Sigma})}
$$

by the sum

$$
(1-x) \sum_{T \in H_{1}(\alpha)} \operatorname{sgn}(T) \sum_{\boldsymbol{\Sigma} \in S_{n,\left\{\alpha_{1}\right\}}^{L}} x^{\operatorname{comdes}_{\beta(T)}(\boldsymbol{\Sigma})} \mathbf{Q}^{i n v(\boldsymbol{\Sigma})} \mathbf{P}^{\operatorname{coinv}(\boldsymbol{\Sigma})}
$$

where $H_{1}(\alpha)$ is the set of special rim hook tabloids of shape $\alpha$ whose first special rim hook is horizontal and $S_{n,\left\{\alpha_{1}\right\}}^{L}$ is the set of all $L$-tuples of permutations $\Sigma$ such that $\alpha_{1} \in \operatorname{Comdes}(\boldsymbol{\Sigma})$. Then we can repeat the same argument on this class of special rim hook tabloids by examining such fillings in pairs with identical integer fillings and identical 
special rim hooks except that one has a break in the special rim hooks at the second row and the other does not. It will follow that we can replace the sum in (4.4) by

$$
(1-x)^{2} \sum_{T \in H_{2}(\alpha)} \operatorname{sgn}(T) \sum_{\boldsymbol{\Sigma} \in S_{n,\left\{\alpha_{1}, \alpha_{1}+\alpha_{2}\right\}}^{L}} x^{\operatorname{comdes}_{\beta(T)}(\boldsymbol{\Sigma})} \mathbf{Q}^{i n v(\boldsymbol{\Sigma})} \mathbf{P}^{\operatorname{coinv}(\boldsymbol{\Sigma})}
$$

where $H_{2}$ is the set of special rim hook tabloids of shape $\alpha$ whose first two special rim hook are horizontal and $S_{n,\left\{\alpha_{1}, \alpha_{1}+\alpha_{2}\right\}}^{L}$ is the set of all $L$-tuples of permutations $\boldsymbol{\Sigma}$ such that $\alpha_{1}, \alpha_{1}+\alpha_{2} \in$ Comdes $(\boldsymbol{\Sigma})$.

Continuing in this way, we can show that if $\alpha$ has $k$ parts, then $[n]_{\mathbf{P}, \mathbf{Q}} ! \xi\left(Z_{\alpha}\right)$ is equal to the sum

$$
(1-x)^{k-1} \sum_{T \in H_{k}(\alpha)} \operatorname{sgn}(T) \sum_{\boldsymbol{\Sigma} \in S_{n, \operatorname{Set}(\alpha)}} x^{\operatorname{comdes}_{\beta(T)}(\boldsymbol{\Sigma})} \mathbf{Q}^{\operatorname{inv}(\boldsymbol{\Sigma})} \mathbf{P}^{\operatorname{coinv}(\boldsymbol{\Sigma})}
$$

where $H_{k}$ is the set of special rim hook tabloids of shape $\alpha$ whose first $\mathrm{k}$ special rim hook are horizontal and $S_{n, \operatorname{Set}(\alpha)}^{L}$ is the set of all $L$-tuples of permutations $\boldsymbol{\Sigma}$ such that $\operatorname{Set}(\alpha) \subseteq \operatorname{Comdes}(\boldsymbol{\Sigma})$. However there is only one special rim hook tabloid $T$ whose first $k$ special rim hooks are horizontal and, clearly, $\operatorname{sgn}(T)=1$ and $\beta(T)=\alpha$. Note that if $\boldsymbol{\Sigma}$ is such that $\operatorname{Set}(\alpha) \subseteq \operatorname{Comdes}(\boldsymbol{\Sigma})$, then $x^{\operatorname{comdes}(\boldsymbol{\Sigma})}=x^{k-1} x^{\operatorname{comdes}_{\alpha}(\boldsymbol{\Sigma})}$. Thus, it follows that

$$
\begin{aligned}
{[n]_{\mathbf{P}, \mathbf{Q}} ! \xi\left(Z_{\alpha}\right) } & =(1-x)^{k-1} \sum_{\boldsymbol{\Sigma} \in S_{n}^{L}, \operatorname{Set}(\alpha) \subseteq \operatorname{Comdes}(\boldsymbol{\Sigma})} x^{\operatorname{comdes}_{\alpha}(\boldsymbol{\Sigma})} \mathbf{Q}^{i n v(\boldsymbol{\Sigma})} \mathbf{P}^{\operatorname{coinv}(\boldsymbol{\Sigma})} \\
& =\frac{(1-x)^{k-1}}{x^{k-1}} \sum_{\boldsymbol{\Sigma} \in S_{n}^{L}, \operatorname{Set}(\alpha) \subseteq \operatorname{Comdes}(\boldsymbol{\Sigma})} x^{\operatorname{comdes}(\boldsymbol{\Sigma})} \mathbf{Q}^{i n v(\boldsymbol{\Sigma})} \mathbf{P}^{\operatorname{coinv}(\boldsymbol{\Sigma})},
\end{aligned}
$$

as desired.

We can then combine Theorem 3.1 with Theorem 4.3 to obtain the following.

Theorem 4.4. Let $\alpha=\left(\alpha_{1}, \ldots, \alpha_{k}\right)$ be a composition of $n$ and let

$$
\overline{\operatorname{Set}}(\alpha)=\left\{\alpha_{1}, \alpha_{1}+\alpha_{2}, \ldots, \alpha_{1}+\alpha_{2}+\cdots+\alpha_{k}\right\} .
$$

Then

$$
\begin{aligned}
\sum_{n \geqslant 1} \frac{t^{n+|\alpha|}}{[n+|\alpha|]_{\mathbf{P}, \mathbf{Q} !}} \sum_{\substack{\boldsymbol{\Sigma}=\left(\sigma^{(1)}, \ldots, \sigma^{(L)}\right) \in S_{n}^{L} \\
\operatorname{Set}(\alpha) \subseteq \operatorname{Comdes}(\boldsymbol{\Sigma})}} x^{\operatorname{comdes}(\boldsymbol{\Sigma})} \mathbf{Q}^{i n v(\boldsymbol{\Sigma})} \mathbf{P}^{\operatorname{coinv}(\boldsymbol{\Sigma})} \\
=\frac{x^{k}}{(1-x)^{k}} \xi\left(\frac{\sum_{j=0}^{k} Z_{\alpha^{(j)}} t^{\left|\alpha^{(j)}\right|}(-1)^{j}}{E(-t)}+\right. \\
\left.(-1)^{k-1}+\sum_{j=1}^{k}(-1)^{j-1} \sum_{r=1}^{\alpha_{j}-1} Z_{\left(\alpha^{(j)}, r\right)} t^{r+\left|\alpha^{(j)}\right|}\right) .
\end{aligned}
$$


Proof. It follows from Theorems 3.1 and 4.3 that (4.5) arises by applying $\xi$ to

$$
\sum_{n \geqslant 1} Z_{(\alpha, n)} t^{n+|\alpha|}
$$

For example, suppose that we want to compute

$$
\sum_{n \geqslant 1} \frac{t^{n+|\alpha|}}{[n+|\alpha|]_{\mathbf{P}, \mathbf{Q}} !} \sum_{\substack{\boldsymbol{\Sigma}=\left(\sigma^{(1)}, \ldots, \sigma^{(L)}\right) \in S_{n}^{L} \\\{2,4\} \subseteq \operatorname{Comdes}(\boldsymbol{\Sigma})}} x^{\operatorname{comdes}(\boldsymbol{\Sigma})} \mathbf{Q}^{i n v(\boldsymbol{\Sigma})} \mathbf{P}^{\operatorname{coinv}(\boldsymbol{\Sigma})}
$$

To have the left-hand side of (4.5) equal (4.6), we must chose $\alpha=(2,2)$. Thus we must compute

$$
\frac{x^{2}}{(1-x)^{2}} \xi\left(\frac{Z_{(2,2)} t^{4}-Z_{(2)} t^{2}+1}{E(-t)}+\left(Z_{(2,1)} t^{3}-\left(Z_{(1)} t+1\right)\right)\right) .
$$

This necessitates computing the expansion of $Z_{(2,2)}, Z_{(2,1)}$, and $Z_{(2)}$ in terms of the elementary symmetric functions. One can easily list the transposed special rim hook tabloids by hand in each case. These are pictured in Figure 11.
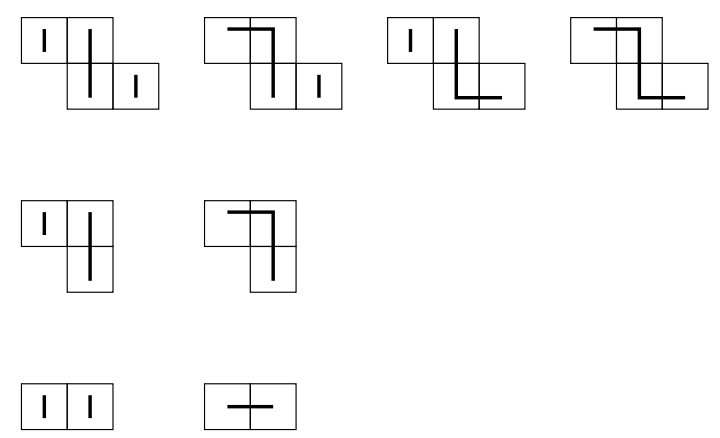

Figure 11: Transposed special rim hook tabloids for $Z_{(2,2)}, Z_{(2,1)}$, and $Z_{2}$.

Thus it follows from (2.1) that

$$
\begin{aligned}
Z_{(2,2)} & =e_{1}^{2} e_{2}-2 e_{1} e_{3}+e_{4}, \\
Z_{(2,1)} & =e_{1} e_{2}-e_{3}, \text { and } \\
Z_{2} & =e_{1}^{2}-e_{2} .
\end{aligned}
$$


Hence,

$$
\begin{aligned}
\frac{x^{2}}{(1-x)^{2}} \xi( & \frac{Z_{(2,2)} t^{4}-Z_{(2)} t^{2}+1}{E(-t)}+\left(Z_{(2,1)} t^{3}-\left(Z_{(1)} t+1\right)\right) \\
= & \frac{x^{2}}{(1-x)^{2} \xi(E(-t))} \xi\left(\left(e_{1}^{2} e_{2}-2 e_{1} e_{3}+e_{4}\right) t^{4}-\left(e_{1}^{2}-e_{2}\right) t^{2}+1\right. \\
& \left.+\left(\left(e_{1} e_{2}-e_{3}\right) t^{3}-e_{1} t-1\right) E(-t)\right) \\
= & \frac{x^{2}}{(1-x)^{2} \xi(E(-t))} \xi\left(\left(e_{1}^{2} e_{2}-2 e_{1} e_{3}+e_{4}\right) t^{4}-\left(e_{1}^{2}-e_{2}\right) t^{2}+1\right. \\
& +\left(\left(e_{1} e_{2}-e_{3}\right) t^{3}-e_{1} t-1\right)\left(1-e_{1} t+e_{2} t^{2}-e_{3} t^{3}\right) \\
& \left.+\left(\left(e_{1} e_{2}-e_{3}\right) t^{3}-e_{1} t-1\right) \sum_{n \geqslant 4} e_{n}(-t)^{n}\right) \\
= & \frac{x^{2}}{(1-x)^{2} \xi(E(-t))} \xi\left(e_{4} t^{4}+\left(e_{1} e_{2}^{2}-e_{2} e_{3}\right) t^{5}+\left(e_{3}^{2}-e_{1} e_{2} e_{3}\right) t^{6}\right. \\
& \left.+\sum_{n \geqslant 4}(-t)^{n+3}\left(e_{3} e_{n}-e_{1} e_{2} e_{n}\right)+\sum_{n \geqslant 4}(-t)^{n+1} e_{1} e_{n}-\sum_{n \geqslant 4} e_{n}(-t)^{n}\right) .
\end{aligned}
$$

Collecting terms, one can show that (4.7) is equal to

$$
\begin{aligned}
& \frac{x^{2}}{(1-x)^{2} \xi(E(-t))} \xi\left((-t)^{5}\left(e_{2} e_{3}-e_{1} e_{2}^{2}+e_{1} e_{4}-e_{5}\right)+\right. \\
& \left.t^{6}\left(e_{3}^{2}-e_{1} e_{2} e_{3}+e_{1} e_{5}-e_{6}\right)+\sum_{n \geqslant 7}(-t)^{n}\left(e_{3} e_{n-3}-e_{1} e_{2} e_{n-3}+e_{1} e_{n-1}-e_{n}\right)\right) \\
& =\frac{x^{2}}{(1-x)^{2} \xi(E(-t))} \xi\left(\sum_{n \geqslant 5}(-t)^{n}\left(e_{3} e_{n-3}-e_{1} e_{2} e_{n-3}+e_{1} e_{n-1}-e_{n}\right)\right) .
\end{aligned}
$$

Now

$$
\begin{aligned}
& \frac{1}{\xi(E(-t))}=\frac{1}{1+\sum_{n \geqslant 1} \frac{\left.(1-x)^{n-1} \mathbf{Q}^{(n} \begin{array}{l}
n \\
2
\end{array}\right)}{[n]_{\mathbf{P}, \mathbf{Q}} !}(-t)^{n}} \\
& =\frac{1-x}{1-x+\sum_{n \geqslant 1} \frac{(1-x)^{n} \mathbf{Q}^{\left(\begin{array}{c}
n \\
2
\end{array}\right)}}{[n]_{\mathbf{P}, \mathbf{Q}} !}(-t)^{n}} \\
& =\frac{1-x}{-x+\exp (t(x-1), \mathbf{P}, \mathbf{Q})} \text {. }
\end{aligned}
$$


Thus it follows that

$$
\begin{aligned}
& \sum_{n \geqslant 1} \frac{t^{n+4}}{[n+4]_{\mathbf{P}, \mathbf{Q}} !} \sum_{\substack{\boldsymbol{\Sigma}=\left(\sigma(1), \ldots, \sigma^{(L)}\right) \in S_{n}^{L} \\
\{2,4\} \subseteq \operatorname{Comdes}(\boldsymbol{\Sigma})}} x^{\operatorname{comdes}(\boldsymbol{\Sigma})} \mathbf{Q}^{\operatorname{inv}(\boldsymbol{\Sigma})} \mathbf{P}^{\operatorname{coinv}(\boldsymbol{\Sigma})} \\
& =\frac{x^{2}}{(1-x)(-x+\exp (t(x-1), \mathbf{P}, \mathbf{Q}))}\left(\sum _ { n \geqslant 5 } ( - t ) ^ { n } \left[\frac{(1-x)^{n-2} \mathbf{Q}^{3+\left(\begin{array}{c}
n-3 \\
2
\end{array}\right)}}{[3]_{\mathbf{P}, \mathbf{Q}} ![n-3]_{\mathbf{P}, \mathbf{Q}} !}\right.\right. \\
& \left.\left.-\frac{(1-x)^{n-3} \mathbf{Q}^{1+\left(\begin{array}{c}
n-3 \\
2
\end{array}\right)}}{[2]_{\mathbf{P}, \mathbf{Q}} ![n-3]_{\mathbf{P}, \mathbf{Q}} !}+\frac{(1-x)^{n-2} \mathbf{Q}^{\left(\begin{array}{c}
n-1 \\
2
\end{array}\right)}}{[n-1]_{\mathbf{P}, \mathbf{Q}} !}-\frac{(1-x)^{n-1} \mathbf{Q}^{\left(\begin{array}{c}
n \\
2
\end{array}\right)}}{[n]_{\mathbf{P}, \mathbf{Q}} !}\right]\right) \\
& =\frac{x^{2}}{(-x+\exp (t(x-1), \mathbf{P}, \mathbf{Q}))}\left(\sum_{n \geqslant 5} \frac{(-t)^{n}(1-x)^{n-2} \mathbf{Q}^{\left(\begin{array}{c}
n-3 \\
2
\end{array}\right)}}{[n]_{\mathbf{P}, \mathbf{Q}} !}\right. \\
& \left.\times\left[-\frac{\mathbf{Q}\left[\begin{array}{c}
n \\
1,2, n-3
\end{array}\right]_{\mathbf{P}, \mathbf{Q}}}{(1-x)^{2}}+\frac{\mathbf{Q}^{3}\left[\begin{array}{c}
n \\
3
\end{array}\right]_{\mathbf{P}, \mathbf{Q}}+\mathbf{Q}^{2 n-5}[n]_{\mathbf{P}, \mathbf{Q}}}{(1-x)}-\mathbf{Q}^{3 n-6}\right]\right) .
\end{aligned}
$$

\section{Extensions}

In this section, we shall make a few remarks about possible extensions of Theorem 4.4. If $\boldsymbol{\Sigma} \in S_{n}^{L}$, we let oneRise $(\boldsymbol{\Sigma})=\{1, \ldots, n-1\}-\operatorname{Comdes}(\boldsymbol{\Sigma})$. Now suppose we given a pair of disjoint sets of finite positive integers $(S, T)$. Then we would like to find the generating

functions over sets of $L$-tuples of permutations $\Sigma \in S_{n}^{L}$ such that $S \subseteq \operatorname{Comdes}(\boldsymbol{\Sigma})$ and $T \subseteq$ oneRise $(\boldsymbol{\Sigma})$. We can easily obtain such generating functions by inclusion-exclusion. That is, consider

$$
\begin{aligned}
& \phi_{(S, T)}(x, \mathbf{P}, \mathbf{Q}) \\
& =\sum_{S \subseteq U \subseteq S \cup T}(-1)^{|U-S|} \sum_{n \geqslant 0} \frac{t^{n}}{[n]_{\mathbf{P}, \mathbf{Q}} !} \sum_{\substack{\boldsymbol{\Sigma} \in S_{n}^{L} \\
U \subseteq \operatorname{Comdes}(\boldsymbol{\Sigma})}} x^{\operatorname{comdes}(\boldsymbol{\Sigma})} \mathbf{Q}^{i n v(\boldsymbol{\Sigma})} \mathbf{P}^{\operatorname{coinv}(\boldsymbol{\Sigma})}
\end{aligned}
$$

where we interpret the sum

$$
\sum_{\substack{\boldsymbol{\Sigma} \in S_{h}^{L} \\ U \subseteq C o m d e s(\boldsymbol{\Sigma})}} x^{\operatorname{comdes}(\boldsymbol{\Sigma})} \mathbf{Q}^{i n v(\boldsymbol{\Sigma})} \mathbf{P}^{\operatorname{coinv}(\boldsymbol{\Sigma})}
$$

to be 0 if there are no $L$-tuples $\Sigma \in S_{n}^{L}$ such that $U \subseteq \operatorname{Comdes}(\Sigma)$. That is, if $n$ is too small, it may be the case that there are no $L$-tuples $\Sigma \in S_{n}^{L}$ such that $U \subseteq \operatorname{Comdes}(\Sigma)$. Then it is easy to see that

$$
\begin{aligned}
\phi_{(S, T)}(x, \mathbf{P}, \mathbf{Q})= & \sum_{n \geqslant 0} \frac{t^{n}}{[n]_{\mathbf{P}, \mathbf{Q}} !} \sum_{\boldsymbol{\Sigma} \in S_{n}^{L}} x^{\operatorname{comdes}(\boldsymbol{\Sigma})} \mathbf{Q}^{i n v(\boldsymbol{\Sigma})} \mathbf{P}^{\operatorname{coinv}(\boldsymbol{\Sigma})} \\
& \times \sum_{S \subseteq U \subseteq S \cup T}(-1)^{|U-S|} \chi(U \subseteq \operatorname{Comdes}(\boldsymbol{\Sigma})) .
\end{aligned}
$$


It then easily follows from inclusion-exclusion that

$$
\sum_{S \subseteq U \subseteq S \cup T}(-1)^{|U-S|} \chi(U \subseteq \operatorname{Comdes}(\boldsymbol{\Sigma}))
$$

is equal to 1 if $S \subseteq \operatorname{Comdes}(\boldsymbol{\Sigma})$ and $T \cap \operatorname{Comdes}(\boldsymbol{\Sigma})=\emptyset$ and is equal to 0 otherwise. Thus

$$
\phi_{(S, T)}(x, \mathbf{P}, \mathbf{Q})=\sum_{\substack{\boldsymbol{\Sigma} \in S_{n}^{L} \\ S \subseteq \operatorname{Comdes}(\boldsymbol{\Sigma}), T \cap \operatorname{Comdes}(\boldsymbol{\Sigma})=\emptyset}} x^{\operatorname{comdes}(\boldsymbol{\Sigma})} \mathbf{Q}^{i n v(\boldsymbol{\Sigma})} \mathbf{P}^{\operatorname{coinv}(\boldsymbol{\Sigma})} .
$$

When $n \geqslant 1+\max (S \cup T)$, the condition $S \subseteq \operatorname{Comdes}(\boldsymbol{\Sigma})$ and $T \cap \operatorname{Comdes}(\boldsymbol{\Sigma})=\emptyset$ is equivalent to the condition that $S \subseteq \operatorname{Comdes}(\boldsymbol{\Sigma})$ and $T \subseteq$ oneRise $(\boldsymbol{\Sigma})$.

For example, suppose that we wish to find the generating function for $L$-tuples of permutations $\boldsymbol{\Sigma}$ such that $\{1,3\} \subseteq \operatorname{Comdes}(\boldsymbol{\Sigma})$ and $\{2\} \subseteq$ oneRise $(\boldsymbol{\Sigma})$. Then

$$
\begin{aligned}
\sum_{n \geqslant 1} \frac{t^{3+n}}{[3+n]_{\mathbf{P}, \mathbf{Q}} !} & \sum_{\substack{\boldsymbol{\Sigma} \in S_{n}^{L} \\
\{1,3\} \subseteq \operatorname{Comdes}(\boldsymbol{\Sigma}) \\
\{2\} \cap \operatorname{Comdes}(\boldsymbol{\Sigma})=\emptyset}} x^{\operatorname{comdes}(\boldsymbol{\Sigma})} \mathbf{Q}^{\operatorname{inv}(\boldsymbol{\Sigma})} \mathbf{P}^{\operatorname{coinv}(\boldsymbol{\Sigma})} \\
= & \sum_{n \geqslant 1} \frac{t^{3+n}}{[3+n]_{\mathbf{P}, \mathbf{Q}} !} \sum_{\substack{\boldsymbol{\Sigma} \in S_{n}^{L} \\
\{1,3\} \subseteq \operatorname{Comdes}(\boldsymbol{\Sigma})}} x^{\operatorname{comdes}(\boldsymbol{\Sigma})} \mathbf{Q}^{\operatorname{inv}(\boldsymbol{\Sigma})} \mathbf{P}^{\operatorname{coinv}(\boldsymbol{\Sigma})} \\
& -\sum_{n \geqslant 1} \frac{t^{3+n}}{[3+n]_{\mathbf{P}, \mathbf{Q}} !} \sum_{\substack{\boldsymbol{\Sigma} \in S_{n}^{L} \\
\{1,2,3\} \subseteq \operatorname{Comdes}(\boldsymbol{\Sigma})}} x^{\operatorname{comdes}(\boldsymbol{\Sigma})} \mathbf{Q}^{\text {inv }(\boldsymbol{\Sigma})} \mathbf{P}^{\operatorname{coinv}(\boldsymbol{\Sigma})} .
\end{aligned}
$$

We can apply Theorem 4.4 with $\alpha=(1,2)$ to conclude that

$$
\begin{aligned}
& \sum_{n \geqslant 1} \frac{t^{3+n}}{[3+n]_{\mathbf{P}, \mathbf{Q} !} !} \sum_{\substack{\boldsymbol{\Sigma} \in S_{n}^{L} \\
\{1,3\} \subseteq \operatorname{Comdes}(\mathbf{\Sigma})}} x^{\operatorname{comdes}(\mathbf{\Sigma})} \mathbf{Q}^{i n v(\boldsymbol{\Sigma})} \mathbf{P}^{\operatorname{coinv}(\boldsymbol{\Sigma})} \\
&= \frac{x^{2}}{(1-x)^{2}} \xi\left(\frac{Z_{(1,2)} t^{3}-Z_{(1)}+1}{E(-t)}+Z_{(1,1)} t^{2}-1\right) \\
&= \frac{x^{2}}{(1-x)^{2} \xi(E(-t))} \xi\left(Z_{(1,2)} t^{3}-Z_{(1)} t+1+\left[Z_{(1,1)} t^{2}-1\right] E(-t)\right) \\
&= \frac{x^{2}}{(1-x)(-x+e x p(t(x-1), \mathbf{P}, \mathbf{Q}))} \xi\left(\left(e_{1} e_{2}-e_{3}\right) t^{3}-e_{1} t+1\right. \\
&\left.+\left[e_{2} t^{2}-1\right]\left(1-e_{1} t+e_{2} t^{2}-e_{3} t^{3}\right)+\left[e_{2} t^{2}-1\right] \sum_{n \geqslant 4}(-t)^{n} e_{n}\right)
\end{aligned}
$$




$$
\begin{aligned}
= & \frac{x^{2}}{(1-x)(-x+\exp (t(x-1), \mathbf{P}, \mathbf{Q}))} \\
& \times \xi\left(e_{2}^{2} t^{4}-e_{2} e_{3} t^{5}+\left[e_{2} t^{2}-1\right] \sum_{n \geqslant 4}(-t)^{n} e_{n}\right) \\
= & \frac{x^{2}}{(1-x)(-x+\exp (t(x-1), \mathbf{P}, \mathbf{Q}))}\left(\frac{(1-x)^{2} \mathbf{Q}^{2}}{\left([2]_{\mathbf{P}, \mathbf{Q}} !\right)^{2}} t^{4}\right. \\
& \left.-\frac{(1-x)^{3} \mathbf{Q}^{4}}{[2]_{\mathbf{P}, \mathbf{Q}} ![3]_{\mathbf{P}, \mathbf{Q}} !} t^{5}+\left[\frac{(1-x) \mathbf{Q}}{[2]_{\mathbf{P}, \mathbf{Q}} !} t^{2}-1\right] \sum_{n \geqslant 4}(-t)^{n} \frac{(1-x)^{n-1} \mathbf{Q}^{\left(\begin{array}{c}
n \\
2
\end{array}\right)}}{[n]_{\mathbf{P}, \mathbf{Q}} !}\right) .
\end{aligned}
$$

Here we have used that $Z_{(2,1)}=e_{1} e_{2}-e_{3}$ and $Z_{(1,1)}=e_{2}$. Similarly, we can apply Theorem 4.4 with $\alpha=(1,1,1)$ to conclude that

$$
\begin{aligned}
\sum_{n \geqslant 1} \frac{t^{3+n}}{[3+n]_{\mathbf{P}, \mathbf{Q}} !} & \sum_{\substack{\boldsymbol{\Sigma} \in S_{n}^{L} \\
\{1,2,3\} \subseteq \operatorname{Comdes}(\boldsymbol{\Sigma})}} x^{\operatorname{comdes}(\boldsymbol{\Sigma})} \mathbf{Q}^{\operatorname{inv}(\boldsymbol{\Sigma})} \mathbf{P}^{\operatorname{coinv}(\boldsymbol{\Sigma})} \\
= & \frac{x^{3}}{(1-x)^{3}} \xi\left(\frac{Z_{(1,1,1)} t^{3}-Z_{(1,1)} t^{2}+Z_{(1)} t-1}{E(-t)}+1\right) \\
= & \frac{x^{3}}{(1-x)^{3} \xi(E(-t))} \xi\left(Z_{(1,1,1)} t^{3}-Z_{(1,1)} t^{2}+Z_{(1)} t-1+E(-t)\right) \\
= & \frac{x^{3}}{(1-x)^{2}(-x+\exp (t(x-1), \mathbf{P}, \mathbf{Q}))} \xi\left(\sum_{n \geqslant 4}(-t)^{n} e_{n}\right) \\
= & \frac{x^{3}}{(1-x)^{2}(-x+\exp (t(x-1), \mathbf{P}, \mathbf{Q}))}\left(\sum_{n \geqslant 4}(-t)^{n} \frac{(1-x)^{n-1} \mathbf{Q}^{\left(\begin{array}{l}
n \\
2
\end{array}\right)}}{\left.[n]_{\mathbf{P}, \mathbf{Q} !}\right)}\right.
\end{aligned}
$$

Subtracting (5.2) from (5.1) and putting the terms over a common denominator, we see that

$$
\begin{aligned}
\sum_{n \geqslant 1} \frac{t^{3+n}}{[3+n]_{\mathbf{P}, \mathbf{Q}} !} & \sum_{\substack{\boldsymbol{\Sigma} \in S_{n}^{L} \\
\{1,3\} \subseteq \operatorname{Comdes}(\boldsymbol{\Sigma}) \\
\{2\} \cap \operatorname{Comdes}(\boldsymbol{\Sigma})=\emptyset}} x^{\operatorname{comdes}(\boldsymbol{\Sigma})} \mathbf{Q}^{\operatorname{inv}(\boldsymbol{\Sigma})} \mathbf{P}^{\operatorname{coinv}(\boldsymbol{\Sigma})} \\
& =\frac{x^{2}}{(1-x)^{2}(-x+\exp (t(x-1), \mathbf{P}, \mathbf{Q}))} R
\end{aligned}
$$


where

$$
\begin{aligned}
& R=\frac{(1-x)^{3} \mathbf{Q}^{2}}{\left([2]_{\mathbf{P}, \mathbf{Q}} !\right)^{2}} t^{4}-\frac{(1-x)^{4} \mathbf{Q}^{4}}{[2]_{\mathbf{P}, \mathbf{Q}} ![3]_{\mathbf{P}, \mathbf{Q}} !} t^{5} \\
& +\frac{(1-x)^{2} \mathbf{Q}}{[2]_{\mathbf{P}, \mathbf{Q}} !} t^{2} \sum_{n \geqslant 4}(-t)^{n} \frac{(1-x)^{n-1} \mathbf{Q}^{\left(\begin{array}{l}
n \\
2
\end{array}\right)}}{[n]_{\mathbf{P}, \mathbf{Q}} !} \\
& -(1-x) \sum_{n \geqslant 4}(-t)^{n} \frac{(1-x)^{n-1} \mathbf{Q}^{\left(\begin{array}{c}
n \\
2
\end{array}\right)}}{[n]_{\mathbf{P}, \mathbf{Q}} !}-x \sum_{n \geqslant 4}(-t)^{n} \frac{(1-x)^{n-1} \mathbf{Q}^{\left(\begin{array}{l}
n \\
2
\end{array}\right)}}{[n]_{\mathbf{P}, \mathbf{Q}} !} \\
& =\frac{(1-x)^{3} \mathbf{Q}^{2}}{\left([2]_{\mathbf{P}, \mathbf{Q}} !\right)^{2}} t^{4}-\frac{(1-x)^{4} \mathbf{Q}^{4}}{[2]_{\mathbf{P}, \mathbf{Q}} ![3]_{\mathbf{P}, \mathbf{Q} !}} t^{5} \\
& +\sum_{n \geqslant 4}(-t)^{n+2} \frac{(1-x)^{n+1} \mathbf{Q}^{1+\left(\begin{array}{l}
n \\
2
\end{array}\right)}}{[2]_{\mathbf{P}, \mathbf{Q}} ![n]_{\mathbf{P}, \mathbf{Q}} !}-\sum_{n \geqslant 4}(-t)^{n} \frac{(1-x)^{n-1} \mathbf{Q}^{\left(\begin{array}{l}
n \\
2
\end{array}\right)}}{[n]_{\mathbf{P}, \mathbf{Q}} !} \\
& =\left(\frac{(1-x)^{3} \mathbf{Q}^{2}}{\left([2]_{\mathbf{P}, \mathbf{Q}} !\right)^{2}}-\frac{(1-x)^{3} \mathbf{Q}^{6}}{[4]_{\mathbf{P}, \mathbf{Q}} !}\right) t^{4} \\
& -\left(\frac{(1-x)^{4} \mathbf{Q}^{4}}{[2]_{\mathbf{P}, \mathbf{Q}} ![3]_{\mathbf{P}, \mathbf{Q}} !}-\frac{(1-x)^{4} \mathbf{Q}^{10}}{[5]_{\mathbf{P}, \mathbf{Q}} !}\right) t^{5} \\
& +\sum_{n \geqslant 6}(-t)^{n}(1-x)^{n-1}\left(\frac{\mathbf{Q}^{1+\left(\begin{array}{c}
n-2 \\
2
\end{array}\right)}}{[2]_{\mathbf{P}, \mathbf{Q}} ![n-2]_{\mathbf{P}, \mathbf{Q}} !}-\frac{\mathbf{Q}^{\left(\begin{array}{l}
n \\
2
\end{array}\right)}}{[n]_{\mathbf{P}, \mathbf{Q}} !}\right) \\
& =\sum_{n \geqslant 4}(-t)^{n} \frac{(1-x)^{n-1} \mathbf{Q}^{1+\left(\begin{array}{c}
n-2 \\
2
\end{array}\right)}}{[n]_{\mathbf{P}, \mathbf{Q}} !}\left(\left[\begin{array}{l}
n \\
2
\end{array}\right]_{\mathbf{P}, \mathbf{Q}}-\mathbf{Q}^{2 n-4}\right) \text {. }
\end{aligned}
$$

Thus we have proved the following.

\section{Theorem 5.1.}

$$
\begin{aligned}
& \sum_{n \geqslant 1} \frac{t^{3+n}}{[3+n]_{\mathbf{P}, \mathbf{Q}} !} \sum_{\substack{\boldsymbol{\Sigma} \in S_{n}^{L} \\
\{1,3\} \subseteq \text { Comdes }(\boldsymbol{\Sigma}) \\
\{2\} \cap \operatorname{Comdes}(\boldsymbol{\Sigma})=\emptyset}} x^{\operatorname{comdes}(\boldsymbol{\Sigma})} \mathbf{Q}^{\operatorname{inv}(\boldsymbol{\Sigma})} \mathbf{P}^{\operatorname{coinv}(\boldsymbol{\Sigma})} \\
& \quad=\frac{x^{2}}{(-x+\exp (t(x-1), \mathbf{P}, \mathbf{Q})} \sum_{n \geqslant 4}(-t)^{n} \frac{(1-x)^{n-3} \mathbf{Q}^{1+\left(\begin{array}{c}
n-2 \\
2
\end{array}\right)}}{[n]_{\mathbf{P}, \mathbf{Q}} !}\left(\left[\begin{array}{c}
n \\
2
\end{array}\right]_{\mathbf{P}, \mathbf{Q}}-\mathbf{Q}^{2 n-4}\right) .
\end{aligned}
$$

We end this section by showing that we can use a different method to compute the generating functions of $L$-tuples of $\boldsymbol{\Sigma}=\left(\sigma^{(1)}, \ldots, \sigma^{(L)}\right) \in S_{n}^{L}$ such that $\{1,3\} \subseteq$ Comdes $(\boldsymbol{\Sigma})$ and $\{2\} \subseteq \operatorname{Comris}(\boldsymbol{\Sigma})$ where $\operatorname{Comris}(\boldsymbol{\Sigma})=\bigcap_{i=1}^{L} \operatorname{Rise}\left(\sigma^{(i)}\right)$. Note that if $L \geqslant 2$, then the condition that $\{2\} \subseteq \operatorname{Comris}(\boldsymbol{\Sigma})$ is different than the condition that $\{2\} \cap \operatorname{Comdes}(\boldsymbol{\Sigma})=\emptyset$ because the latter condition only asserts the $\{2\} \in \operatorname{Rise}\left(\sigma^{(i)}\right)$ for some $i$ rather than all $i$.

The basic idea is the following. We can apply the reasoning from Theorem 4.1 to

$$
[n]_{\mathbf{P}, \mathbf{Q}} ! \xi\left(h_{n}\right)=\sum_{\mathcal{O} \in \mathcal{L} F((n))} W(\mathcal{O})
$$


where $\mathcal{L} F(n)$ denotes the set of all labeled filled brick tabloids $T=\left(b_{1}, \ldots, b_{k}\right)$ of shape $(n)$ such that the permutations are decreasing within each brick. Now we would like to modify things so that the following conditions are met.

1. the first brick $b_{1}$ is of length 4 or greater,

2. each permutation $\sigma^{(i)}$ has the property that $\sigma^{(i)}(1)>\sigma^{(i)}(2)<\sigma^{(i)}(3)>\sigma^{(i)}(4)>$ $\cdots>\sigma^{(i)}\left(b_{1}\right)$ and $\sigma^{(i)}$ decreases in the remaining bricks, and

3. the labels on cells 1 and 3 are $x$, the label on cell 2 is 1 , and the remaining labels are as before.

We can accomplish this by replacing $h_{n}$ in (5.5) by $p_{n}^{\vec{u}}$ for an appropriate $\vec{u}$. To this end, assume $n \geqslant 4$ and let $T_{n}$ be the set of permutations $\sigma \in S_{n}$ such that $\sigma(1)>\sigma(2)<$ $\sigma(3)>\sigma(4)>\sigma(5)>\cdots>\sigma(n)$. That is, $T_{n}$ is the set of permutations such that $\operatorname{Rise}(\sigma)=\{2\}$. We want to compute

$$
\sum_{\sigma \in T_{n}} q^{i n v(\sigma)} p^{\operatorname{coinv}(\sigma)}
$$

Now if $\sigma \in T_{n}$, then either (i) $\sigma(3)=n$, or (ii) $\sigma(3)=n-1$, in which case $\sigma(1)$ is forced to be $n$. In case (i), $n$ gives rise to $n-3$ inversions and 2 coinversions. The rest of the permutation consists of two decreasing sequences, one of length 2 and one of length $n-3$ so that by (4.1), these give rise to a factor of $q^{\left(\begin{array}{c}2 \\ 2\end{array}\right)+\left(\begin{array}{c}n-3 \\ 2\end{array}\right)}\left[\begin{array}{c}n-1 \\ 2\end{array}\right]_{p, q}$. Thus the total contribution from case (i) to (5.6) is $q^{1+\left(\begin{array}{c}n-2 \\ 2\end{array}\right)} p^{2}\left[\begin{array}{c}n-1 \\ 2\end{array}\right]_{p, q}$. In case (ii) $n$ gives rise to $n-1$ inversions and no coinversions and $n-1$ gives rise to $n-3$ inversions and 1 coinversion. The result of the permutation consists of two decreasing sequences, one of length 1 and one of length $n-3$ so that by (4.1), these give rise to a factor of $q^{\left(\begin{array}{c}1 \\ 2\end{array}\right)+\left(\begin{array}{c}n-3 \\ 2\end{array}\right)}\left[\begin{array}{c}n-2 \\ 1\end{array}\right]_{p, q}=q^{\left(\begin{array}{c}n-3 \\ 2\end{array}\right)}[n-2]_{p, q}$. Thus the total contribution from case (i) to (5.6) is $q^{n-1+\left(\begin{array}{c}n-2 \\ 2\end{array}\right)} p[n-2]$. Thus

$$
\sum_{\sigma \in T_{n}} q^{i n v(\sigma)} p^{\operatorname{coinv}(\sigma)}=q^{1+\left(\begin{array}{c}
n-2 \\
2
\end{array}\right)} p^{2}\left[\begin{array}{c}
n-1 \\
2
\end{array}\right]_{p, q}+q^{n-1+\left(\begin{array}{c}
n-2 \\
2
\end{array}\right)} p[n-2]_{p, q}
$$

It follows that

$$
\begin{aligned}
\sum_{\boldsymbol{\Sigma}=\left(\sigma^{(1)}, \ldots, \sigma^{(L)}\right) \in T_{n}^{L}} & \mathbf{Q}^{i n v(\boldsymbol{\Sigma})} \mathbf{P}^{\operatorname{coinv}(\boldsymbol{\Sigma})} \\
& =\prod_{i=1}^{L}\left(q_{i}^{1+\left(\begin{array}{c}
n-2 \\
2
\end{array}\right)} p_{i}^{2}\left[\begin{array}{c}
n-1 \\
2
\end{array}\right]_{p_{i}, q_{i}}+q_{i}^{n-1+\left(\begin{array}{c}
n-2 \\
2
\end{array}\right)} p_{i}[n-2]_{p_{i}, q_{i}}\right) \\
& =\mathbf{Q}^{1+\left(\begin{array}{c}
n-2 \\
2
\end{array}\right)}\left(\prod_{i=1}^{L} p_{i}^{2}\left[\begin{array}{c}
n-1 \\
2
\end{array}\right]_{p_{i}, q_{i}}+q_{i}^{n-2} p_{i}[n-2]_{p_{i}, q_{i}}\right) .
\end{aligned}
$$


This given, consider the sequence $\vec{u}=\left(u_{1}, u_{2}, \ldots\right)$ where

$$
u_{n}=\frac{x^{2} \mathbf{Q}^{-\left(\begin{array}{c}
n \\
2
\end{array}\right)}}{(1-x)^{3}} \mathbf{Q}^{1+\left(\begin{array}{c}
n-2 \\
2
\end{array}\right)}\left(\prod_{i=1}^{L} p_{i}^{2}\left[\begin{array}{c}
n-1 \\
2
\end{array}\right]_{p_{i}, q_{i}}+q_{i}^{n-2} p_{i}[n-2]_{p_{i}, q_{i}}\right) .
$$

if $n \geqslant 4$ and $u_{1}=u_{2}=u_{3}=0$. Then we have the following theorem.

Theorem 5.2. For $n \geqslant 4$,

$$
[n]_{\mathbf{P}, \mathbf{Q}} ! \xi\left(p_{n}^{\vec{u}}\right)=\sum_{\substack{\boldsymbol{\Sigma}=\left(\sigma^{(1)}, \ldots, \sigma^{(L)}\right) \in S_{n}^{L} \\ \forall i\left(\sigma^{(i)}(1)>\sigma^{(i)}(2)<\sigma^{(i)}(3)>\sigma^{(i)}(4)\right)}} x^{\operatorname{comdes}(\boldsymbol{\Sigma})} \mathbf{Q}^{i n v(\boldsymbol{\Sigma})} \mathbf{P}^{\operatorname{coinv}(\boldsymbol{\Sigma})} .
$$

Proof. In this case, we shall consider only brick tabloids of shape $(n)$ so we shall write $T=\left(b_{1}, \ldots, b_{\ell(\mu)}\right)$ if the size of the bricks in $T$ are $b_{1}, \ldots, b_{\ell(\mu)}$, reading from left to right. We interpreted $p_{n}^{\vec{u}}$ as the sum over weighted brick tabloids $T=\left(b_{1}, \ldots, b_{\ell}\right)$ where the weight of $T$ is given by $W(T)=u_{b_{\ell}} \prod_{i} e_{b_{i}}$. That is, we weighted the last brick by $u_{b_{\ell}}$. However, by the simple process of reversing the order of the bricks in each brick tabloid, we can also interpret $p_{n}^{\vec{u}}$ as the sum over weighted brick tabloids $T=\left(b_{1}, \ldots, b_{\ell}\right)$ where the weight of $T$ is $W(T)=u_{b_{1}} \prod_{i} e_{b_{i}}$. That is, we can decide to weight the first brick by $u_{b_{1}}$ rather than weight the last brick by $u_{b_{\ell}}$. It follows that

$$
\begin{aligned}
{[n]_{\mathbf{P}, \mathbf{Q}} ! \xi\left(p_{n}^{\vec{u}}\right)=} & {[n]_{\mathbf{P}, \mathbf{Q}} ! \sum_{\mu \vdash n}(-1)^{n-\ell(\mu)} W\left(B_{\mu,(n)}\right) \xi\left(e_{\mu}\right) } \\
= & {[n]_{\mathbf{P}, \mathbf{Q}} ! \sum_{\mu \vdash n}(-1)^{n-\ell(\mu)} \sum_{T=\left(b_{1}, \ldots, b_{\ell(\mu)}\right) \in \mathcal{B}_{\mu,(n)}} u_{b_{1}} \prod_{i=1}^{\ell(\mu)} \frac{(1-x)^{b_{i}-1}}{\left[b_{i}\right]_{\mathbf{P}, \mathbf{Q} !}} } \\
= & \sum_{\mu \vdash n} \sum_{T=\left(b_{1}, \ldots, b_{\ell(\mu)}\right) \in \mathcal{B}_{\mu,(n)}}\left[\begin{array}{c}
n \\
b_{1} \geqslant 4
\end{array}, \ldots, b_{\ell(\mu)}\right]_{\mathbf{P}, \mathbf{Q}} \mathbf{Q}^{\sum_{i=1}^{\ell(\mu)}\left(\begin{array}{c}
b_{i} \\
2
\end{array}\right)}(x-1)^{n-\ell(\mu)} \\
& \times \frac{x^{2} \mathbf{Q}^{-\left(\begin{array}{c}
b_{1} \\
2
\end{array}\right)}}{(1-x)^{3}} \mathbf{Q}^{1+\left({ }^{b_{1}-2}{ }_{2}\right)}\left(\prod_{i=1}^{L} p_{i}^{2}\left[\begin{array}{c}
b_{1}-1 \\
2
\end{array}\right]_{p_{i}, q_{i}}+q_{i}^{b_{1}-2} p_{i}\left[b_{1}-2\right]_{p_{i}, q_{i}}\right) .
\end{aligned}
$$

As in the proof of Theorem 4.1, for any $T=\left(b_{1}, \ldots, b_{\ell(\mu)}\right) \in \mathcal{B}_{\mu,(n)}$, we can interpret

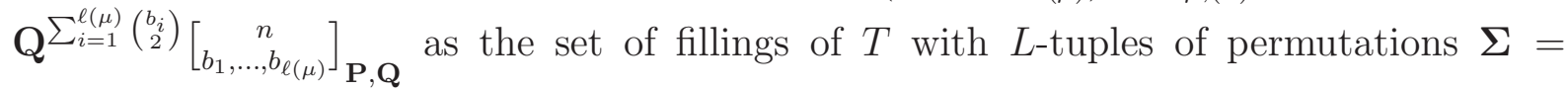
$\left(\sigma^{(1)}, \ldots, \sigma^{(L)}\right)$ such that for each $i$, the elements of $\sigma^{(i)}$ are decreasing within each brick of $T$, and we weight such a filling with $\mathbf{Q}^{i n v(\boldsymbol{\Sigma})} \mathbf{P}^{\operatorname{coinv}(\boldsymbol{\Sigma})}$. There is a factor of $x^{2}(x-1)^{n-\ell(\mu)-3}$ in (5.8) arising from $T$, which we interpret as taking such a filling and labeling cells 1 and 3 with $x$, labeling cell 2 with 1 , and labeling each remaining cell which is not at the end of a brick with either $x$ or -1 , and labeling each cell at the end of a brick with 1 . Finally, given (5.7), we can interpret the extra factor,

$$
\mathbf{Q}^{-\left(\begin{array}{c}
b_{1} \\
2
\end{array}\right)} \mathbf{Q}^{1+\left(\begin{array}{c}
b_{1}-2 \\
2
\end{array}\right)}\left(\prod_{i=1}^{L} p_{i}^{2}\left[\begin{array}{c}
b_{1}-1 \\
2
\end{array}\right]_{p_{i}, q_{i}}+q_{i}^{b_{1}-2} p_{i}\left[b_{1}-2\right]_{p_{i}, q_{i}}\right)
$$


as allowing us to replace the decreasing fillings of the first brick by all fillings where in each row $\sigma^{(i)}(1)>\sigma^{(i)}(2)<\sigma^{(i)}(3)>\sigma^{(i)}(4)$. That is, by rearranging the elements in the first brick, we do not change the number of inversions and coinversions between elements that lie in the first brick and the rest of elements in any given row. Thus, we need only account for the difference between the inversions and coinversions for elements that lie in the first brick caused by going from a decreasing sequence in each row to a sequence such that

$$
\sigma^{(i)}(1)>\sigma^{(i)}(2)<\sigma^{(i)}(3)>\sigma^{(i)}(4)>\sigma^{(i)}(5)>\cdots>\sigma^{(i)}\left(b_{1}\right) .
$$

Clearly, when all the elements that lie in the first brick form a decreasing sequence in each row, they contribute a factor of $\mathbf{Q}^{\left(\begin{array}{c}b_{1} \\ 2\end{array}\right)}$ to $\mathbf{Q}^{i n v(\boldsymbol{\Sigma})} \mathbf{P}^{\operatorname{coinv}(\boldsymbol{\Sigma})}$. After we arrange the sequences, the elements in the first brick contribute

$$
\mathbf{Q}^{1+\left({ }^{b_{1}-2}\right)}\left(\prod_{i=1}^{L} p_{i}^{2}\left[\begin{array}{c}
b_{1}-1 \\
2
\end{array}\right]_{p_{i}, q_{i}}+q_{i}^{b_{1}-2} p_{i}\left[b_{1}-2\right]_{p_{i}, q_{i}}\right)
$$

to $\mathbf{Q}^{i n v(\boldsymbol{\Sigma})} \mathbf{P}^{\operatorname{coinv}(\boldsymbol{\Sigma})}$. Thus the factor

$$
\left.\mathbf{Q}^{-\left(\begin{array}{c}
b_{1} \\
2
\end{array}\right)} \mathbf{Q}^{1+\left({ }^{b_{1}-2}\right)}\right)\left(\prod_{i=1}^{L} p_{i}^{2}\left[\begin{array}{c}
b_{1}-1 \\
2
\end{array}\right]_{p_{i}, q_{i}}+q_{i}^{b_{1}-2} p_{i}\left[b_{1}-2\right]_{p_{i}, q_{i}}\right)
$$

is exactly what we need to account for replacing the decreasing sequences in each row of the first brick by sequences such that

$$
\sigma^{(i)}(1)>\sigma^{(i)}(2)<\sigma^{(i)}(3)>\sigma^{(i)}(4)>\sigma^{(i)}(5)>\cdots>\sigma^{(i)}\left(b_{1}\right) .
$$

We shall call an object $\mathcal{O}$ created in this way a did-labeled filled brick tabloid where did is short for decrease-increase-decrease. An example of a did-labeled filled brick tabloid for $L=3$ and $n=12$ is given in Figure 12. Then we define the weight of $\mathcal{O}, W(\mathcal{O})$, to be the product over all the labels of the cells times $\mathbf{Q}^{i n v(\boldsymbol{\Sigma})} \mathbf{P}^{\operatorname{coinv}(\boldsymbol{\Sigma})}$ if $T$ is filled with permutations $\boldsymbol{\Sigma}=\left(\sigma^{(1)}, \ldots \sigma^{(L)}\right)$. Thus for the object pictured in Figure 12,

$$
W(\mathcal{O})=(-1)^{4} x^{4} q_{1}^{\operatorname{inv}\left(\sigma^{(1)}\right)} q_{2}^{\operatorname{inv}\left(\sigma^{(2)}\right)} q_{3}^{\operatorname{inv}\left(\sigma^{(3)}\right)} p_{1}^{\operatorname{coinv}\left(\sigma^{(1)}\right)} p_{2}^{\operatorname{coinv}\left(\sigma^{(2)}\right)} p_{3}^{\operatorname{coinv}\left(\sigma^{(3)}\right)} .
$$

We let $\mathcal{D} I D L F(n)$ denote the set of all objects that can be created in this way from brick tabloid $T$ of shape $(n)$. Then it follows that

$$
[n]_{\mathbf{P}, \mathbf{Q}} ! \xi\left(p_{n}^{\vec{u}}\right)=\sum_{\mathcal{O} \in \mathcal{D} I D L F(n)} W(\mathcal{O})
$$

Then we define an involution $I: \mathcal{D} I D L F(n) \rightarrow \mathcal{D} I D L F(n)$ exactly as before. That is, given $\mathcal{O} \in \mathcal{D} I D L F(n)$, read the cells of $\mathcal{O}$ from left to right and look for the first cell $c$ such that either

(i) $c$ is labeled with -1 or 


\begin{tabular}{|c|c|c|c|c|c|c|c|c|c|c|c|}
\hline $\mathbf{x}$ & 1 & $\mathbf{x}$ & -1 & 1 & -1 & $\mathbf{x}$ & 1 & -1 & -1 & $\mathbf{X}$ & 1 \\
\hline 10 & 6 & 9 & 4 & 1 & 11 & 7 & 3 & 12 & 8 & 5 & 2 \\
\hline 9 & 1 & 6 & 3 & 2 & 10 & 7 & 5 & 12 & 11 & 8 & 4 \\
\hline 12 & 5 & 10 & 6 & 4 & 9 & 3 & 1 & 11 & 8 & 7 & 2 \\
\hline
\end{tabular}

\begin{tabular}{|c|c|c|c|c|c|c|c|c|c|c|}
\hline$\sigma^{(1)}=$ & 6 & 9 & 4 & 1 & 11 & 7 & 3 & 12 & 8 & 5 \\
\hline$\sigma^{(2)}=$ & 1 & 6 & 3 & 2 & 10 & 7 & 5 & 12 & 11 & 8 \\
\hline$\sigma^{(3)}=12$ & 5 & 10 & 6 & 4 & 9 & 3 & 1 & 11 & 8 & 7 \\
\hline
\end{tabular}

Figure 12: A did-labeled filled brick tabloid of shape (12).

(ii) $c$ is at the end of end of brick $b$, the cell $c+1$ is immediately to the right of $c$ and starts another brick $b^{\prime}$, and each permutation $\sigma^{(i)}$ decreases as we go from $c$ to $c+1$.

If we are in case $(\mathrm{i})$, then $I(\mathcal{O})$ is the did-labeled filled brick tabloid which is obtained from $\mathcal{O}$ by taking the brick $b$ that contains $c$ and splitting $b$ into two bricks $b_{1}$ and $b_{2}$ where $b_{1}$ contains the cells of $b$ up to and including the cell $c$ and $b_{2}$ contains the remaining cells of $b$, and changing the label on $c$ from -1 to 1 . In case (ii), $I(\mathcal{O})$ is the did-labeled filled brick tabloid which is obtained from $\mathcal{O}$ by combining the two bricks $b$ and $b^{\prime}$ into a single brick and changing the label on cell $c$ from 1 to -1 . Finally, if neither case (i) nor case (ii) applies, then we let $I(\mathcal{O})=\mathcal{O}$. For example, for the labeled filled brick tabloid $\mathcal{O}$ pictured in Figure 12, I(O) is pictured in Figure 13.

\begin{tabular}{|c|c|c|c|c|c|c|c|c|c|c|c|}
\hline $\mathbf{X}$ & 1 & $\mathbf{X}$ & 1 & 1 & -1 & $\mathbf{X}$ & 1 & -1 & -1 & $\mathbf{X}$ & $\mathbf{1}$ \\
\hline 10 & 6 & 9 & 4 & 1 & 11 & 7 & 3 & 12 & 8 & 5 & 2 \\
\hline 9 & 1 & 6 & 3 & 2 & 10 & 7 & 5 & 12 & 11 & 8 & 4 \\
\hline 12 & 5 & 10 & 6 & 4 & 9 & 3 & 1 & 11 & 8 & 7 & 2 \\
\hline
\end{tabular}

Figure 13: $I(\mathcal{O})$.

As before, $I$ is a weight-preserving sign-reversing involution, so that

$$
\begin{aligned}
{[n]_{\mathbf{P}, \mathbf{Q}} ! \xi\left(p_{n}^{\vec{u}}\right) } & =\sum_{\mathcal{O} \in \mathcal{D} \operatorname{IDLF}(n)} W(\mathcal{O}) \\
& =\sum_{\mathcal{O} \in \mathcal{D I D L F}(n), I(\mathcal{O})=\mathcal{O}} W(\mathcal{O}) .
\end{aligned}
$$

Thus we must examine the fixed points of $I$. Clearly if $I(\mathcal{O})=\mathcal{O}$, then $\mathcal{O}$ can have no cells which are labeled with -1 . Also it must be the case that between any two consecutive bricks of $\mathcal{O}$, at least one of the underlying permutations $\sigma^{(i)}$ must increase. It follows 
that each cell $c$ which is not among the first three cells and which is not at the end of the brick in $\mathcal{O}$ is labeled with $x$ and each of the permutation $\sigma^{(i)}$ has a descent at $c$ so that $c \in \operatorname{Comdes}(\boldsymbol{\Sigma})$. All other cells of $\mathcal{O}$ are either at the end of brick which has another brick to its right, in which case $c \notin \operatorname{Comdes}(\boldsymbol{\Sigma})$. All such cells have label 1 so that $W(\mathcal{O})=x^{\operatorname{comdes}(\boldsymbol{\Sigma})} \mathbf{Q}^{\operatorname{inv}(\boldsymbol{\Sigma})} \mathbf{P}^{\operatorname{coinv}(\boldsymbol{\Sigma})}$.

Now, if we are given $\boldsymbol{\Sigma}=\left(\sigma^{(1)}, \ldots, \sigma^{(L)}\right) \in S_{n}^{L}$ such that for each $i, \sigma^{(i)}(1)>\sigma^{(i)}(2)<$ $\sigma^{(i)}(3)>\sigma^{(i)}(4)$, then we can construct of fixed point of $I$ from $\boldsymbol{\Sigma}$ by using $\left(\sigma^{(1)}, \ldots, \sigma^{(L)}\right)$ to fill a tabloid of shape $(n)$ and drawing the bricks so that the cells $c$ which end bricks are precisely the elements of oneRise $(\boldsymbol{\Sigma})$ which are greater than 3 . This shows that

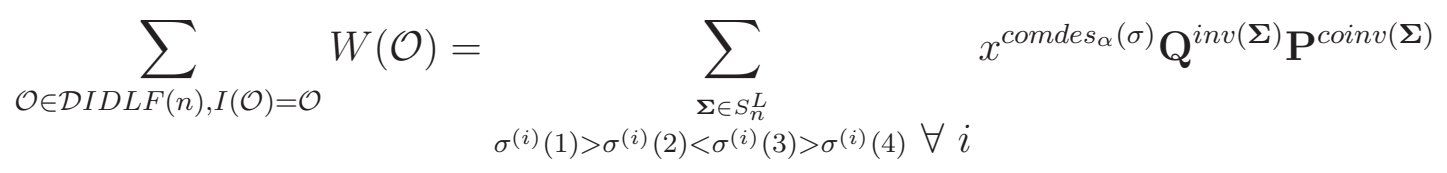

as desired.

Using Theorem 5.2, we can apply $\xi$ to both sides of the identity

$$
\sum_{n \geqslant 1} p_{n}^{\vec{u}} t^{n}=\frac{\sum_{n \geqslant 1}(-1)^{n-1} u_{n} e_{n} t^{n}}{E(-t)}
$$

to prove the following:

$$
\begin{aligned}
& \sum_{n \geqslant 1} \frac{t^{3+n}}{[3+n]_{\mathbf{P}, \mathbf{Q}} !} \sum_{\substack{\boldsymbol{\Sigma} \in S_{3+n}^{L} \\
\forall i\left(\sigma^{(i)}(1)>\sigma^{(i)}(2)<\sigma^{(i)}(3)>\sigma^{(i)}(4)\right)}} x^{\operatorname{comdes}_{\alpha}(\Sigma)} \mathbf{Q}^{i n v(\boldsymbol{\Sigma})} \mathbf{P}^{\operatorname{coinv}(\boldsymbol{\Sigma})} \\
& =\frac{x^{2}}{(1-x)^{2}(-x+\exp (t(x-1), \mathbf{P}, \mathbf{Q}))} \\
& \times \sum_{n \geqslant 4}(-1)^{n-1} \frac{(1-x)^{n-1} \mathbf{Q}^{\left(\begin{array}{l}
n \\
2
\end{array}\right)}}{[n]_{\mathbf{P}, \mathbf{Q}} !} \mathbf{Q}^{-\left(\begin{array}{c}
n \\
2
\end{array}\right)} \mathbf{Q}^{1+\left(\begin{array}{c}
n-2 \\
2
\end{array}\right)} \\
& \times\left(\prod_{i=1}^{L} p_{i}^{2}\left[\begin{array}{c}
b_{1}-1 \\
2
\end{array}\right]_{p_{i}, q_{i}}+q_{i}^{b_{1}-2} p_{i}\left[b_{1}-2\right]_{p_{i}, q_{i}}\right) \\
& =\frac{x^{2}}{(-x+\exp (t(x-1), \mathbf{P}, \mathbf{Q}))} \\
& \times \sum_{n \geqslant 4}(-1)^{n-1} \frac{(1-x)^{n-3}}{[n]_{\mathbf{P}, \mathbf{Q}} !} \mathbf{Q}^{1+\left(\begin{array}{c}
n-2 \\
2
\end{array}\right)}\left(\prod_{i=1}^{L} p_{i}^{2}\left[\begin{array}{c}
b_{1}-1 \\
2
\end{array}\right]_{p_{i}, q_{i}}+q_{i}^{b_{1}-2} p_{i}\left[b_{1}-2\right]_{p_{i}, q_{i}}\right) .
\end{aligned}
$$

We note that it should be the case that (5.4) and (5.8) are equal when $L=1$ since the condition that $2 \notin \operatorname{Comdes}(\boldsymbol{\Sigma})$ and $2 \in \operatorname{ComRise}(\boldsymbol{\Sigma})$ is the same in that case. Indeed, 
the two expressions are the same as

$$
\begin{aligned}
p_{1}^{2}\left[\begin{array}{c}
n-1 \\
2
\end{array}\right]_{p_{1}, q_{1}}+q_{1}^{n-2} p_{1}[n-2]_{p_{1}, q_{1}} & =\frac{[n-1]_{p_{1}, q_{1}} ![n-2]_{p_{1}, q_{1}} !}{[2]_{p_{1}, q_{1}} !}+q_{1}^{n-2}\left([n-1]_{p_{1}, q_{1}}-q_{1}^{n-2}\right) \\
& =\frac{[n-1]_{p_{1}, q_{1}} !}{[2]_{p_{1}, q_{1}} !}\left(p_{1}^{2}[n-2]_{p_{1}, q_{1}}+q_{1}^{n-2}[2]_{p_{1}, q_{1}}\right)-q_{1}^{2 n-4} \\
& =\frac{[n-1]_{p_{1}, q_{1}} !}{[2]_{p_{1}, q_{1}} !}[n]_{p_{1}, q_{1}}-q_{1}^{2 n-4}-q_{1}^{2 n-4} \\
& =\left[\begin{array}{c}
n \\
2
\end{array}\right]_{p_{1}, q_{1}}-q_{1}^{2 n-4} .
\end{aligned}
$$

We note that our second method can be applied to obtain generating functions for any $L$-tuple of permutations $\boldsymbol{\Sigma}$ which are to contain a given set $S$ of common descents and a given set $T$ of common rises where $S \cup T=\{1, \ldots, n\}$. That is, let $\mathcal{D}_{n+k}^{S, T}$ equal the set of permutations $\sigma \in S_{n+k}$ such that $S \cup\{n+1, \ldots, n+k-1\} \subseteq \operatorname{Des}(\sigma)$ and $T \subseteq \operatorname{Rise}(\sigma)$ and suppose that we can compute

$$
D_{n+k}^{S, T}(p, q)=\sum_{\sigma \in \mathcal{D}_{n+k}^{S, T}} q^{i n v(\sigma)} p^{\operatorname{coinv}(\sigma)} .
$$

Then we can define our sequence $\vec{u}=\left(u_{1}, u_{2}, \ldots\right)$ so that

(1) $u_{i}=0$ if $i \leqslant n$ and

(2) $u_{i}=\frac{x^{|S|}}{(1-x)^{n}} \prod_{i=1}^{L} D_{n+k}^{S, T}\left(p_{i}, q_{i}\right)$.

Then the same type of argument that was used to prove (5.8) will prove that

$$
[n+k]_{\mathbf{P}, \mathbf{Q}} ! \xi\left(p_{n+k}^{\vec{u}}\right)=\sum_{\substack{\boldsymbol{\Sigma} \in S_{n+k}^{L} \\ S \subseteq \operatorname{Comdes}(\boldsymbol{\Sigma}) \\ T \subseteq \operatorname{ComRise}(\boldsymbol{\Sigma})}} x^{\operatorname{comdes}(\boldsymbol{\Sigma})} \mathbf{Q}^{\operatorname{inv}(\boldsymbol{\Sigma})} \mathbf{P}^{\operatorname{coinv}(\boldsymbol{\Sigma})}
$$

Finally, we should note that in a forthcoming paper, we shall show how we can combine the two methods described in this paper. The key idea is to define an analogue of the ribbon Schur function $Z_{\alpha}$ in terms of special rim hook tabloids in such that way that $T$ is a special rim hook tabloid of shape $F_{(\alpha, n)}$ whose special rim hooks have length $a_{1}, \ldots, a_{k}$, reading from top to bottom. Then we will weight $T$ by $\operatorname{sgn}(T) p_{a_{1}}^{\vec{u}} h_{a_{2}} \cdots h_{a_{k}}$ instead of $\operatorname{sgn}(T) h_{a_{1}} h_{a_{2}} \cdots h_{a_{k}}$.

The authors would like to thank the anonymous referee for a very careful reading of the paper and suggestions that improved the presentation of the paper. 


\section{References}

[1] D. Beck, J. Remmel, and T. Whitehead, The combinatorics of transition matrices between the bases of the symmetric functions and the $B_{n}$ analogues, Discrete Mathematics 153 (1996), 3-27.

[2] F. Brenti, Permutation enumeration, symmetric functions, and unimodality, Pacific J. Math. 157 (1993), 1-28.

[3] F. Brenti, A class of q-symmetric functions arising from plethysm, J. Comb. Th. Ser. A, 91 (2000), 137-170.

[4] L. Carlitz, Sequences and inversions, Duke Math. J. 37 (1970), 193-198.

[5] Ö. Eg̃eciog̃lu and J.B. Remmel, A Combinatorial interpretation of the Inverse Kostka Matrix, Linear and Multilinear Algebra, 26, (1990), pp 59-84.

[6] Ö. Eg̃eciog̃lu and J. Remmel, Brick tabloids and connection matrices between bases of symmetric functions, Discrete Applied Math. 34 (1991), 107-120.

[7] J-M. Fédou and D. Rawlings, Statistics on pairs of permutations, Discrete Math. 143 (1995), 31-45.

Adjacencies in words, Adv. Appl. Math. 16 (1995), 206-218.

[8] T.M. Langley, Alternative transition matrices for Brenti's q-symmetric functions and a class of $q, t$-symmetric functions on the hyperoctahedral group, Proceedings of the 2002 Conference on Formal Power Series and Algebraic Combinatorics, Melbourne Australia.

[9] T.M. Langley and J.B. Remmel, Enumeration of $m$-tuples of permuations and a new class of power bases for the space of symmetric functions, Advances in App. Math. 36 (2006), 30-66.

[10] I. G. MacDonald, "Symmetric Functions and Hall Polynomials," Oxford Univ. Press, London/New York, 1979.

[11] A. Mendes, Building generating functions brick by brick, Ph.D. thesis, University of California, San Diego, 2004.

[12] A. Mendes and J.B. Remmel, Permutations and words counted by consecutive patterns, preprint.

[13] A. Mendes and J.B. Remmel, Generating Functions from Symmetric Functions, preprint.

[14] D. Ram, J.B. Remmel, and T. Whitehead, Combinatorics of the q-basis of symmetric functions, J. Comb. Th. Series A, 76(2) (1996), 231-271.

[15] R. P. Stanley, Binomial posets, Möbius inversion and permuation enumeration, J. Comb. Th. (A), 20 (1976), 336-356.

[16] Jennifer D. Wagner, The permutation enumeration of wreath products and cyclic and symmetric groups, Advances in Appl. Math. 30 (2003), 343-368. 